\title{
Approval Voting on Dichotomous Preferences
}

\author{
Marc Vorsatz*
}

September 7, 2004

\begin{abstract}
The aim of this paper is to find normative foundations of Approval Voting. In order to show that Approval Voting is the only social choice function that satisfies anonymity, neutrality, strategy-proofness and strict monotonicity we rely on an intermediate result which relates strategy-proofness of a social choice function to the properties of Independence of Irrelevant Alternatives and monotonicity of the corresponding social welfare function. Afterwards we characterize Approval Voting by means of strict symmetry, neutrality and strict monotonicity and relate this result to May's Theorem [11]. Finally, we show that it is possible to substitute the property of strict monotonicity by the one efficiency of in the second characterization.
\end{abstract}

Keywords: Approval Voting, Dichotomous Preferences, Social Choice Function, Social Welfare Function.

JEL-Number: D71.

\section{Introduction}

Given a set of individuals with preferences on alternatives, a set-valued social choice function or social choice correspondence selects for all preferences profiles a subset of alternatives. Yet, there are socio-economic environments where alternatives are incompatible and a unique alternative has to be selected. In such kind of situations we can give a meaning to set-valued social choice functions by interpreting the image as a preselected set from which the winning

\footnotetext{
*I thank Jordi Massó for his supervision and his never ending encouragement. Coralio Ballester, Salvador Barberà, Dolors Berga, Carmen Beviá, Bhaskar Dutta, Lars Ehlers, Alejandro Neme, Hervé Moulin, Shmuel Nitzan and Yves Sprumont helped me a lot with their comments. This research was undertaken with support from the fellowship 2001FI 00451 of the Generalitat de Catalunya and from the research grant BEC2002-02130 of the Ministerio de Ciencia y Tecnología of Spain.

Address of contact: Departament d'Economia i d'Història Econòmica, Universitat Autònoma de Barcelona, Edifici B, 08193 Bellaterra, Spain. E-Mail: mvorsatz@idea.uab.es
} 
alternative has still to be determined. The main objective of this paper is to study set-valued social choice functions axiomatically when individuals have dichotomous preferences on the set of alternatives and believe that all preselected alternatives have the same chance of being finally chosen. ${ }^{1}$

To be more concrete, we are interested in the following kind of problem: Consider a firm which opens a job offer for specialized candidates with a certain profile. Since the amount of extractable information from the applications is partial, firms use different filters before taking their final decision. The first one is often to invite a number of candidates for a job interview. If the recruiting committee decides by voting whom to interview, then every member of the recruiting committee classifies candidates either as "acceptable" or as "nonacceptable", that is the members of recruiting committee have dichotomous preference on the set of candidates. Thus, we ask how the different opinions should be aggregated in order to determine whom to invite for a job interview.

Yet, it can happen that only the opinion of a subgroup of individuals is available, because some members of the recruiting committee may strictly prefer to abstain from voting for reasons which lie outside of the model. Similarly, some candidates may have already accepted job offers from other firms, and, as a result, the recruiting committee faces a restricted choice set. In order to capture these two restriction in our model we consider families of set-valued social choice functions instead of set-valued social choice functions. The drawback of the more general analysis is that the image of the family can be conditionalized on the set of feasible alternatives and the set of voters. To rule out this possibility, we impose two consistency conditions that describe how the set of preselected alternatives changes as the set of feasible alternatives or the set of voters varies. The condition regarding the set of voters states that individuals who are indifferent between all feasible alternatives do not matter for the final outcome, whereas the one regarding the set of feasible alternatives strengthens the rationalizability condition property $\alpha$ of Sen and Pattanaik [14]. ${ }^{2}$ Finally,

\footnotetext{
${ }^{1}$ Among others, further work on set-valued social choice functions is due to Duggan and Schwartz [7], Barberà et. al [1], and Ching and Zhou [6].

${ }^{2}$ Property $\alpha$ states that if one compares two social choice correspondences differing from each other in such a way that the considered set of alternatives in the second problem is a
} 
we define a social choice rule to be a family of set-valued social choice functions that is consistent in alternatives and individuals, and therefore, the question we deal with is which social choice rule satisfies a set of desirable properties.

One key property to be investigated is strategy-proofness, because it formalizes the idea that individuals have incentives to represent their preferences truthfully. ${ }^{3}$ Since individuals have dichotomous preferences on the set of alternative and, given a set of feasible alternatives and a set of voters, the social choice function is set-valued, we have to make assumptions how individual order subsets of alternatives in order to introduce of strategy-proofness properly. To see this suppose that the set of alternatives is equal to $\{x, y, z\}$ and that the dichotomous preference relation for some individual is such that $\{z\}$ is the only non-acceptable alternative. In this case, the ordering between $\{x\}$ and the set $\{x, y, z\}$ is not defined which implies that we cannot make statement regarding incentives. To solve this problem we extend the dichotomous preferences in such a way as if individuals believed that the final step of the decision process is resolved by a lottery which gives equal chance to every preselected alternative. This extension is called cohesive.

In the third Section of the paper, we analyze the relationship between families of set-valued social choice functions and social welfare functions. Blair and Muller [3] show that a domain of strict preferences admits the construction of a strategy-proof family of single-valued social choice functions if and only if the domain admits the construction of a social welfare function which is monotone and satisfies Independence of Irrelevant Alternatives (IIA). A similar result is not true for dichotomous preferences when preferences are extended in a cohesive way. In particular, strategy-proofness on the cohesive dichotomous domain of a family of set-valued social choice functions is a necessary but not a suf-

subset of the one considered in the first problem, then every alternative in the image of the first social choice correspondence has to be in the image of the second one. We assume that the choice set of the second problem is equal to the choice set of the first one restricted to the set of alternatives which is still available.

${ }^{3}$ The most important contribution on incentive problems in social choice theory is the impossibility theorem of Gibbard and Satterthwaite which has been extended to social choice correspondences by Duggan and Schwartz [7]. Given its negative flavor, one strand of the literature examines the existence of strategy-proof social choice functions on restricted domains, e.g. Grove and Loeb [10], Moulin [12], Sprumont [15], and Barberà et. al [2]. 
ficient condition for the corresponding social welfare function to be monotone and to satisfy IIA. Proposition 1 states that it is possible to recover sufficiency by imposing in addition to strategy-proofness the properties of neutrality and consistency in individuals.

Approval Voting is one of the most prominent voting rules. Organizations which apply it are diverse and include the United Nations, the Mathematical Association of America, the Econometric Society, and the Institute for Operations Research and Management Sciences. ${ }^{4}$ In their seminal paper Brams and Fishburn [5] propose a preference extension that together with the definition of stragegy-proofness induces the same incentives on the dichotomous preference domain as cohesive preferences. Then they show that Approval Voting is strategy-proof on the extended preference domain and selects the set of Condorcet winners. Recently Vorsatz [16] has shown that the Borda Count is equivalent to Approval Voting on the dichotomous preference domain and that all scoring rules different from the Borda Count are manipulable on the cohesive dichotomous domain. Hence, three of the most well known aggregation rules coincide on the dichotomous preference domain which makes Approval Voting an intuitive choice. To our knowledge the only characterizations of Approval Voting on the dichotomous preference domain are due to Fishburn [8] and [9]. In [9] the preference extension proposed in the paper by Brams and Fishburn [5] is used in order to show that Approval Voting is the only family of anonymous set-valued social choice function which is neutral, strategy-proof, and satisfies consistency property. On the other hand, in [8] it is shown that if the set of alternatives contains at least three alternatives, then Approval Voting is characterized among all anonymous set-valued social choice functions by means of neutrality, consistency and disjoint equality. In Section 4, we make use of the results of Section 3 in order to prove that a social choice strategy-proofness on the cohesive dichotomous domain, neutrality, anonymity and strict monotonicity if and only if it is Approval Voting (Theorem 1). This results differs from the characterization of Fishburn [8] mainly in three points: First, we use

\footnotetext{
${ }^{4}$ See a recent article of Brams [4] who describes the success of Approval Voting in a number of organizations.
} 
the property of strict monotonicity instead of a consistency condition. Second, in Theorem 1 anonymity is a proper axiom, and finally, Fishburn [8] considers the set of voters to be fixed. From this point we restrict the analysis to the case when all individuals reveal their preferences. Theorem 2 and 3 state that a social choice function is strict symmetry, neutrality, and strict monotonicity (efficient) if and only if it is Approval Voting. ${ }^{5}$ Since strict symmetry strengthens the property of anonymity, we can relate Theorem 2 to May's Theorem [11] which states that if the number of alternatives is equal to two and the preference domain is the set of all weak orders on the set of alternatives, then the Majority Rule is characterized by means of anonymity, neutrality, and strict monotonicity. Hence, Theorem 2 shows that it is possible to consider any number of alternatives if the property of anonymity is replaced by the one of strict symmetry.

The remainder of the paper is organized as follows. In the next Section, we introduce notation and basic definitions. In Section 3, the results which relate social welfare functions and social choice functions are presented. In Section 4, we propose three different characterizations of Approval Voting. Some additional results and examples can be found in the Appendix.

\section{Notation and Definitions}

Consider a group of individuals $N$ with preferences on the set of alternatives $K$ whose objective is to aggregate their preferences by choosing a non-empty subset of $K$. It is assumed that individuals can abstain from voting, that is only the set of individuals $\bar{N} \subseteq N$ participates in the decision process. Moreover, it may happen that only a subset of alternatives is feasible, and therefore, we restrict the set of implementable alternatives to be equal to $\bar{K} \subseteq K$. The aggregation problem is interesting only if $|\bar{K}| \equiv \bar{k} \geq 2$ and $|\bar{N}| \equiv \bar{n} \geq 1$.

\footnotetext{
${ }^{5}$ The intuition of strict symmetry is as follows: Suppose that there are two different preference profile which differ from each other just because some alternative which is good for the first individual and bad the second individual according to first preference profile is good for the second individual and bad for the first individual according to the second preference profile. Then, the image of a social choice function is the same at both preference profiles.
} 
Let $R_{i}$ be the weak preference relation of individual $i$ on $K$. The strict and the indifference preference relations associated with $R_{i}$ are denoted by $P_{i}$ and $I_{i}$, respectively. The set of all weak preferences on $K$ is denoted by $\mathcal{R}$. A domain $\overline{\mathcal{R}}$ is a subset of $\mathcal{R}$. Given a set of individuals $\bar{N} \subseteq N$ and a domain $\overline{\mathcal{R}} \subseteq \mathcal{R}$, a preference profile $R_{\bar{N}}=\left(R_{i}\right)_{i \in \bar{N}} \in \overline{\mathcal{R}}^{\bar{N}}$ is a vector of individual preference relations. The role of individual $i$ in the preference profile $R_{\bar{N}} \in \overline{\mathcal{R}}^{\bar{N}}$ is emphasized by writing $R_{\bar{N}}=\left(R_{i}, R_{\bar{N} \backslash\{i\}}\right)$.

The preference relation $R_{i}$ is dichotomous if it consists of up to two indifference classes which are called the set of good alternatives and the set of bad alternatives. Given $R_{i} \in \overline{\mathcal{R}}$, define the set of good alternatives associated with $R_{i}$ as $G\left(R_{i}\right)=\left\{g \in K: g R_{i} y\right.$ for all $\left.y \in K\right\}$. Similarly, let $B\left(R_{i}\right)=\{b \in K$ : $y R_{i} b$ for all $\left.y \in K\right\}$ be the set of bad alternatives corresponding to $R_{i}$. The cardinalities of the two sets are given by $g\left(R_{i}\right)$ and $b\left(R_{i}\right)$. Then, $R_{i} \in \mathcal{R}$ is dichotomous if and only if $G\left(R_{i}\right) \cup B\left(R_{i}\right)=K$. The domain of all dichotomous preferences is denoted by $\mathcal{D} \subset \mathcal{R}$. Let $D_{i} \in \mathcal{D}$ be a particular dichotomous preference relation for individual $i$. Finally, given a set of individuals $\bar{N} \subseteq N$ and a preference profile $\left(D_{i}\right)_{i \in \bar{N}} \in \mathcal{D}^{\bar{N}}$, let $N_{x}\left(D_{\bar{N}}\right)=\left|\left\{i \in \bar{N}: x \in G\left(D_{i}\right)\right\}\right|$ be the support of $x$ at $D_{\bar{N}}$.

A family of social choice functions $\left\{f^{\bar{K}, \bar{N}}: \mathcal{D}^{\bar{N}} \rightarrow 2^{\bar{K} \backslash\{\emptyset\}}\right\}_{\bar{K} \subseteq K, \bar{N} \subset N}$ selects for all sets of feasible alternatives $\bar{K} \subseteq K$, all sets of individuals $\bar{N} \subseteq N$, and all preference profiles $D_{\bar{N}} \in \mathcal{D}^{\bar{N}}$ (notice that individuals report their preferences on $K$ and not on $\bar{K})$ a non-empty set of feasible alternatives $f^{\bar{K}, \bar{N}}\left(D_{\bar{N}}\right) \in 2^{\bar{K}} \backslash\{\emptyset\} .{ }^{6}$ With a slight abuse of notation we write $f^{\bar{K}}\left(D_{\bar{N}}\right)$ instead of $f^{\bar{K}, \bar{N}}\left(D_{\bar{N}}\right)$. Throughout indexes are suppressed whenever no restriction is made on the set of feasible alternatives or the set of individuals.

We impose two consistency conditions on the family $\left\{f^{\bar{K}, \bar{N}}\right\}_{\bar{K} \subseteq K, \bar{N} \subseteq N}$ that

\footnotetext{
${ }^{6}$ We do not consider the empty set, because we want to study exclusively situations where some decision has to be taken. Moreover, there are two technical reasons for excluding the empty set: First, since we are interested in strategic voting, we have to make assumptions on how an individual orders subsets of $K$. Yet, if $D_{i} \in \mathcal{D}$ is such that $G\left(D_{i}\right) \neq \emptyset$ and $B\left(D_{i}\right) \neq \emptyset$, then it is not obvious how an individual orders the empty set versus some non-empty subsets of $K$. For example, if $x \in G\left(D_{i}\right)$ and $y \in B\left(D_{i}\right)$, then it is ambiguous whether $i$ prefers the empty set or $\{x, y\}$. Second, Approval Voting is not efficient any more if the empty set can be chosen.
} 
keep track on how the selected set of alternatives varies due to changes in the set of feasible alternatives and the set of individuals. The family of social choice functions $\left\{f^{\bar{K}, \bar{N}}: \mathcal{D}^{\bar{N}} \rightarrow 2^{\bar{K}} \backslash\{\emptyset\}\right\}_{\bar{K} \subseteq K, \bar{N} \subseteq N}$ is said to be consistent in alternatives if for all sets $S \subset T \subseteq K$ and $\bar{N} \subseteq N$, and all preference profiles $D_{\bar{N}} \in \mathcal{D}^{\bar{N}}$, $f^{S}\left(D_{\bar{N}}\right)=f^{T}\left(D_{\bar{N}}\right) \cap S$ whenever $f^{T}\left(D_{\bar{N}}\right) \cap S \neq \emptyset$. Observe that consistency in alternatives is a stronger rationalizability condition than the one proposed by Sen and Pattanaik [14] (property $\alpha$ ) which asks that $f^{T}\left(D_{\bar{N}}\right) \cap S \subseteq f^{S}\left(D_{\bar{N}}\right)$. The meaning of this condition is as follows: The decision makers suppose a priori that every alternative is feasible and determine which alternatives to pre-select. After taking this decision it may turn out that less alternatives are feasible, and therefore, the set of pre-selected alternative is restricted accordingly. Given the sets $A \subset C \subseteq N$ and the preference profile $D_{C} \in \mathcal{D}^{C}$, denote by $\left.D_{C}\right|_{A} \in \mathcal{D}^{A}$ the preference profile which is obtained by restricting $D_{C} \in \mathcal{D}^{C}$ to $A$. The family of social choice functions $\left\{f^{\bar{K}, \bar{N}}: \mathcal{D}^{\bar{N}} \rightarrow 2^{\bar{K}} \backslash\{\emptyset\}\right\}_{\bar{K} \subseteq K, \bar{N} \subseteq N}$ is said to be consistent in individuals if for all sets $\bar{K} \subseteq K$ and $A \subset C \subseteq N$, and all pairs of preference profiles $D_{A} \in \mathcal{D}^{A}$ and $D_{C} \in \mathcal{D}^{C}$ which are such that (a) $D_{A}=\left.D_{C}\right|_{A}$ and (b) for all $i \in C \backslash A, D_{i}$ satisfies either $G\left(D_{i}\right)=\emptyset$ or $G\left(D_{i}\right)=\bar{K}$, then the condition $f^{\bar{K}}\left(D_{A}\right)=f^{\bar{K}}\left(D_{C}\right)$ holds. Hence, individuals who are indifferent between all feasible alternatives cannot alter the result. Finally, a social choice rule is a family of social choice functions that is consistent in alternatives and individuals. One particular social choice rule is Approval Voting. According to it all alternatives with the highest support are selected.

Definition 1 The social choice rule $\left\{f^{\bar{K}, \bar{N}}: \mathcal{D}^{\bar{N}} \rightarrow 2^{\bar{K}} \backslash\{\emptyset\}\right\}_{\bar{K} \subset K, \bar{N} \subset N}$ is said to be Approval Voting if for all $\bar{K} \subseteq K, \bar{N} \subseteq N$ and $D_{\bar{N}} \in \mathcal{D}^{\bar{N}}, x \in f^{\bar{K}}\left(D_{\bar{N}}\right)$ if and only if $N_{x}\left(D_{\bar{N}}\right) \geq N_{y}\left(D_{\bar{N}}\right)$ for all $y \in \bar{K}$.

Example 1: Denote the generic social choice function $f^{\bar{K}, \bar{N}}$ associated with Approval Voting by $f_{A}^{\bar{K}, \bar{N}}$. Next, consider the special case when $N=\{1,2,3\}$, $K=\{x, y, z\}$, and the preference profile $D \in \mathcal{D}^{N}$ is such that $G\left(D_{1}\right)=\{x, y\}$, $G\left(D_{2}\right)=\{x, z\}$ and $G\left(D_{3}\right)=\{y\}$. In this case, $f_{A}(D)=\{x, y\}, f_{A}^{\{y, z\}}(D)=$ $\{y\}$, and $f_{A}^{\{x, y\}}\left(D_{\{1,2\}}\right)=\{x\}$. 
One of our aims is to concentrate on social choice functions that provide incentives for individuals to represent their preferences truthfully. Since $f^{\bar{K}, \bar{N}}$ is a set-valued function, we have to make assumptions on how individuals extend their orderings on $K$ to non-empty subsets of $K$. One particular way of extending the preference relation $D_{i} \in \mathcal{D}$ to the family of all non-empty subsets of $K$ is to assume that individuals evaluate the set $S \subseteq K$ according to the percentage of good alternatives contained in $S$.

Definition 2 The preference relation $\succsim_{D_{i}}$ on $2^{K} \backslash\{\emptyset\}$ is cohesive with respect to $D_{i}$ whenever for all $S, T \in 2^{K} \backslash\{\emptyset\}, S \succsim_{D_{i}} T$ if and only if $\frac{\left|G\left(D_{i}\right) \cap S\right|}{|S|} \geq \frac{\left|G\left(D_{i}\right) \cap T\right|}{|T|}$ ( $\succsim_{D_{i}}$ is strict whenever the inequality is strict).

The following example illustrates the concept of cohesive preferences.

Example 2: Let the preference relation $D_{i}$ be such that $G\left(D_{i}\right)=\{x, y\}$ and $B\left(D_{i}\right)=\{z\}$. Then, the cohesive preference relation $\succsim_{D_{i}}$ is equal to $\{x\} \sim_{D_{i}}$ $\{y\} \sim_{D_{i}}\{x, y\} \succ_{D_{i}}\{x, y, z\} \succ_{D_{i}}\{x, z\} \sim_{D_{i}}\{y, z\} \succ_{D_{i}}\{z\}$.

The cohesive extension of dichotomous preferences has been rationalized by Vorsatz [16] in the following way: If we interpret $f^{\bar{K}}\left(D_{\bar{N}}\right)$ as a set of pre-selected alternatives from which a unique winning alternative has to be determined via a lottery and individuals are expected utility maximizers, then individuals care only about the probability that a good alternative is chosen. If, in addition, individuals assign to all alternatives belonging to $f^{\bar{K}}\left(D_{\bar{N}}\right)$ the same winning probability (the lottery is neutral), then the lottery with support on $S$ is weakly preferred to the lottery with support on $T$ if and only if $S \succsim_{D_{i}} T$.

Now it is straightforward to define strategy-proofness. Given $\bar{K} \subseteq K$ and $\bar{N} \subseteq N$, the social choice function $f^{\bar{K}, \bar{N}}: \mathcal{D}^{\bar{N}} \rightarrow 2^{\bar{K}} \backslash\{\emptyset\}$ is said to be manipulable by $i$ if for some $D_{\bar{N}} \in \mathcal{D}^{\bar{N}}$ and $D_{i}^{\prime} \in \mathcal{D}, f^{\bar{K}}\left(D_{i}^{\prime}, D_{\bar{N} \backslash\{i\}}\right) \succ_{D_{i}} f^{\bar{K}}\left(D_{\bar{N}}\right)$.

Definition 3 The social choice rule $\left\{f^{\bar{K}, \bar{N}}: \mathcal{D} \rightarrow 2^{K} \backslash\{\emptyset\}\right\}_{\bar{K} \subseteq K, \bar{N} \subseteq N}$ is said to be strategy-proof on the cohesive dichotomous domain if for all $\bar{K} \subseteq K$ and all $\bar{N} \subseteq N, f^{\bar{K}, \bar{N}}$ is not manipulable by any individual.

Anonymity and neutrality formalize the democratic idea that all individuals 
have the same voting power and that there does not exist any alternative which is a priori favored.

Definition 4 The social choice rule $\left\{f^{\bar{K}, \bar{N}}: \mathcal{D}^{\bar{N}} \rightarrow 2^{\bar{K}} \backslash\{\emptyset\}\right\}_{\bar{K} \subseteq K, \bar{N} \subseteq N}$ is said to be anonymous if for all sets $\bar{K} \subseteq K$ and $\bar{N} \subseteq N$, all preference profiles $D_{\bar{N}} \in \mathcal{D}^{\bar{N}}$, and all permutations $\sigma: \bar{N} \rightarrow \bar{N}, f^{\bar{K}}\left(D_{\sigma(\bar{N})}\right)=f^{\bar{K}}\left(D_{\bar{N}}\right)$.

Let $\mu\left(D_{\bar{N}}\right)$ be the vector of dichotomous preference relations for the set of individuals $\bar{N}$ which is obtained by permuting alternatives according to the one-to-one mapping $\mu: K \rightarrow K$.

Definition 5 The social choice rule $\left\{f^{\bar{K}, \bar{N}}: \mathcal{D}^{\bar{N}} \rightarrow 2^{\bar{K}} \backslash\{\emptyset\}\right\}_{\bar{K} \subseteq K, \bar{N} \subseteq N}$ is said to be neutral if for all sets $\bar{K} \subseteq K$ and $\bar{N} \subseteq N$, all preference profiles $\bar{D}_{\bar{N}} \in \mathcal{D}^{\bar{N}}$, and all permutations $\mu: K \rightarrow K, f^{\bar{K}}\left(\mu\left(D_{\bar{N}}\right)\right)=\mu\left(f^{\bar{K}}\left(D_{\bar{N}}\right)\right)$.

The last property to be introduced is strict monotonicity. It can be motivated by interpreting the set $f^{\bar{K}}\left(D_{\bar{N}}\right)$ of size bigger than one as a situation where a tie occurs among all alternatives belonging to $f^{\bar{K}}\left(D_{\bar{N}}\right)$. Then, the tie is broken in favor of a subset $S$ of $f^{\bar{K}}\left(D_{\bar{N}}\right)$ whenever alternatives in $S$ receive additional support by some individual everything else unchanged.

Definition 6 The social choice rule $\left\{f: \mathcal{D}^{\bar{N}} \rightarrow 2^{\bar{K}} \backslash\{\emptyset\}\right\}_{\bar{K} \subseteq K, \bar{N} \subseteq N}$ is said to be strictly monotone if for all sets $S \subseteq \bar{K} \subseteq K, S \neq \emptyset$ and $\bar{N} \subseteq N$, and all pairs of preference profiles $D_{\bar{N}}, D_{\bar{N}}^{\prime} \in \mathcal{D}^{\bar{N}}$ which are such that for some $i$, $G\left(D_{i}\right)=G\left(D_{i}^{\prime}\right) \cup S, G\left(D_{i}^{\prime}\right) \cap S=\emptyset$, and $D_{\bar{N} \backslash\{i\}}=D_{\bar{N} \backslash\{i\}}^{\prime}$, the condition $S \subseteq f^{\bar{K}}\left(D_{\bar{N}}^{\prime}\right)$ implies $f^{\bar{K}}\left(D_{\bar{N}}\right)=S$.

In order to characterize Approval Voting by means of the described properties we build on an intermediate result that relates social choice functions and social welfare functions on the domain of dichotomous preferences. Given a set of individuals $\bar{N} \subseteq N$, a social welfare function $F^{\bar{N}}: \mathcal{D}^{\bar{N}} \rightarrow \mathcal{R}$ selects for all preference profiles $D_{\bar{N}} \in \mathcal{D}^{\bar{N}}$ a complete and transitive social preference relation $F^{\bar{N}}\left(D_{\bar{N}}\right)$ on $K$ which is denoted by $R\left(D_{\bar{N}}\right)$ whenever there is no danger of confusion. The asymmetric and the symmetric part of $R\left(D_{\bar{N}}\right)$ are denoted by $P\left(D_{\bar{N}}\right)$ 
and $I\left(D_{\bar{N}}\right)$, respectively. Given $\bar{K} \subseteq K$ and a preference profile $D_{\bar{N}} \in \mathcal{D}^{\bar{N}}$, let $\left.R\left(D_{\bar{N}}\right)\right|_{\bar{K}}$ be the social ordering restricted to $\bar{K}$. Define the set of top alternatives according to $\left.R\left(D_{\bar{N}}\right)\right|_{\bar{K}}$ as $\left.R^{1}\left(D_{\bar{N}}\right)\right|_{\bar{K}}=\left\{x \in \bar{K}: x R\left(D_{\bar{N}}\right) y\right.$ for all $y \in$ $\bar{K}\}$. Let $N\left(D_{\bar{N}} ; x, y\right)$ be the set of individuals who weakly prefer alternative $x$ to $y$ at $D_{\bar{N}} \in \mathcal{D}^{\bar{N}}$, that is $N\left(D_{\bar{N}} ; x, y\right)=\left\{i \in \bar{N}: x \in G\left(D_{i}\right)\right.$ or $\left.x, y \in B\left(D_{i}\right)\right\}$. Using the former notation we introduce next some properties of social welfare functions. Given $\bar{N} \subseteq N$, the social welfare function $F^{\bar{N}}: \mathcal{D}^{\bar{N}} \rightarrow \mathcal{R}$ satisfies Independence of Irrelevant Alternatives (IIA) if for all pairs of preference profiles $D_{\bar{N}}, D_{\bar{N}}^{\prime} \in \mathcal{D}^{\bar{N}}$ which are such that for some pair of alternatives $(x, y), N\left(D_{\bar{N}} ; x, y\right)=N\left(D_{\bar{N}}^{\prime} ; x, y\right)$ and $N\left(D_{\bar{N}} ; y, x\right)=N\left(D_{\bar{N}}^{\prime} ; y, x\right)$, then $\left.R\left(D_{\bar{N}}\right)\right|_{\{x, y\}}=\left.R\left(D_{\bar{N}}^{\prime}\right)\right|_{\{x, y\}}$. Given $\bar{N} \subseteq N$, the social welfare function $F^{\bar{N}}: \mathcal{D}^{\bar{N}} \rightarrow \mathcal{R}$ is said to be monotone if for all pairs of preference profiles $D_{\bar{N}}, D_{\bar{N}}^{\prime} \in \mathcal{D}^{\bar{N}}$ which are such that for some $i$ and some pair of alternatives $(x, y)$ the conditions $i \notin N\left(D_{\bar{N}} ; x, y\right), i \in N\left(D_{\bar{N}}^{\prime} ; x, y\right)$ and $D_{\bar{N} \backslash\{i\}}^{\prime}=D_{\bar{N} \backslash\{i\}}$ hold, then $x R\left(D_{\bar{N}}\right) y$ implies $x R\left(D_{\bar{N}}^{\prime}\right) y$ (the social welfare function is strictly monotone whenever the former conditions imply that $\left.x P\left(D_{\bar{N}}^{\prime}\right) y\right)$. Given $\bar{N} \subseteq N$, the social welfare function $F^{\bar{N}}: \mathcal{D}^{\bar{N}} \rightarrow \mathcal{R}$ is said to be anonymous if for all $D_{\bar{N}} \in \mathcal{D}^{\bar{N}}$ and all permutations $\sigma: \bar{N} \rightarrow \bar{N}, R\left(D_{\sigma(\bar{N})}\right)=R\left(D_{\bar{N}}\right)$. A family of social welfare functions is a set of functions $\left\{F^{\bar{N}}: \mathcal{D}^{\bar{N}} \rightarrow \mathcal{R}\right\}_{\bar{N} \subseteq N}$.

In order to study the relation between social welfare functions and families of social choice functions it is necessary to prescribe how one is derived from the other. Following Blair and Muller [3], given a family of social choice functions, we define the derived social welfare function pair by pair. That is, given $\bar{N} \subseteq N$ and $\left\{f^{\bar{K}, \bar{N}}: \mathcal{D}^{\bar{N}} \rightarrow 2^{\bar{K}} \backslash\{\emptyset\}\right\}_{\bar{K} \subseteq K}$, the derived social welfare function $F_{f}^{\bar{N}}$ is as follows: For all $x, y \in K$ and $D_{\bar{N}} \in \mathcal{D}^{\bar{N}}, x R_{f}\left(D_{\bar{N}}\right) y$ if and only if $x \in f^{\{x, y\}}\left(D_{\bar{N}}\right)$. In the other direction, given a social welfare function, the derived family of social choice functions is obtained by selecting the set of top alternatives restricted to the set of feasible alternatives. Formally, given $\bar{N} \subseteq N$ and $F^{\bar{N}}: \mathcal{D}^{\bar{N}} \rightarrow \mathcal{R}$, the derived family of social choice functions $\left\{f_{F}^{\bar{K}, \bar{N}}: \mathcal{D}^{\bar{N}} \rightarrow 2^{\bar{K}} \backslash\{\emptyset\}\right\}_{\bar{K} \subseteq K}$ is as follows: For all $\bar{K} \subseteq K$ and $D_{\bar{N}} \in \mathcal{D}^{\bar{N}}$, $f_{\bar{F}}^{\bar{K}}\left(D_{\bar{N}}\right)=\left.R^{1}\left(D_{\bar{N}}\right)\right|_{\bar{K}}$. 


\section{Relating SCF and SWF}

Blair and Muller [3] analyze the relationship between strategy-proof families of social choice functions and social welfare functions that satisfy IIA. They show that (1) if the strict preference domain $\overline{\mathcal{P}} \subset \mathcal{P}$ permits the construction of a strategy-proof family of social choice functions $\left\{f^{\bar{K}}: \overline{\mathcal{P}}^{N} \rightarrow \bar{K}\right\}_{\bar{K} \subseteq K}$, then the derived social welfare function $F_{f}: \overline{\mathcal{P}}^{N} \rightarrow \mathcal{P}$ is monotone and satisfies IIA, and (2) if the strict preference domain $\overline{\mathcal{P}} \subset \mathcal{P}$ permits the construction of a monotone social welfare function $F: \overline{\mathcal{P}}^{N} \rightarrow \mathcal{P}$ that satisfies IIA, then the derived family of social choice functions $\left\{f_{F}^{\bar{K}}: \overline{\mathcal{P}}^{N} \rightarrow \bar{K}\right\}_{\bar{K} \subseteq K}$ is strategyproof. In this Section, we analyze whether it is possible to derive similar results for the dichotomous preference domain. The next example shows that strategyproofness on the cohesive dichotomous domain is not sufficient for the derived social welfare function to satisfy IIA.

Example 3: The family $\left\{g^{\bar{K}, \bar{N}}: \mathcal{D}^{\bar{N}} \rightarrow 2^{\bar{K}} \backslash\{\emptyset\}\right\}_{\bar{K} \subseteq K, \bar{N} \subseteq N}$ is as follows: For all sets $\bar{K} \subseteq K$ and $\bar{N} \subseteq N$, and all preference profiles $D_{\bar{N}} \in \mathcal{D}^{\bar{N}}$, if $x \in f_{A}^{\bar{K}}\left(D_{\bar{N}}\right)$ and there is an $i \in \bar{N}$ whose preference relation $D_{i}$ is such that either $G\left(D_{i}\right)=$ $K$ or $G\left(D_{i}\right)=\emptyset$, then $g^{\bar{K}}\left(D_{\bar{N}}\right)=\{x\}$. Otherwise, $g^{\bar{K}}\left(D_{\bar{N}}\right)=f_{A}^{\bar{K}}\left(D_{\bar{N}}\right)$.

It is easy to see that the family $\left\{g^{\bar{K}, \bar{N}}\right\}_{\bar{K} \subseteq K, \bar{N} \subseteq N}$ is strategy-proof on the cohesive dichotomous domain, because no individual has incentives either to vote for a bad alternative or not to vote for a good alternative. To show that the derived social welfare function does not satisfy IIA for some $\bar{N} \subseteq N$ suppose that $K=\{x, y, z\}$ and $N=\{1,2,3\}$. Let the preference profiles $D, D^{\prime} \in \mathcal{D}^{N}$ be such that $G\left(D_{1}\right)=\{x, y\}, G\left(D_{1}^{\prime}\right)=K, G\left(D_{2}\right)=G\left(D_{2}^{\prime}\right)=\{x\}$, and $G\left(D_{3}\right)=$ $G\left(D_{3}^{\prime}\right)=\{y\}$. In this case, $g^{\{x, y\}}(D)=\{x, y\}$ and $g^{\{x, y\}}\left(D^{\prime}\right)=\{x\}$. If the derived social welfare function $F_{g}$ satisfied IIA, then $\left.R(D)\right|_{\{x, y\}}=\left.R\left(D^{\prime}\right)\right|_{\{x, y\}}$, because all individuals have the same preferences on $\{x, y\}$ at the two preference profiles. Apply the definition of the derived social welfare function $F_{g}$ to see that $x P_{g}(D) y$ and $y R_{g}\left(D^{\prime}\right) x$. Hence, the derived social welfare function $F_{g}$ violates IIA.

In the former example the family $\left\{g^{\bar{K}, \bar{N}}\right\}_{\bar{K} \subseteq K, \bar{N} \subseteq N}$ fails to satisfy consis- 
tency in individuals and neutrality. Proposition 1 states that the derived social welfare function is monotone and satisfies IIA whenever these two properties are satisfied in addition to strategy-proofness on the cohesive dichotomous domain. Moreover, a similar result as the second one of Blair and Muller [3] stated above holds for the dichotomous preference domain.

Proposition 1 (1) If the family of social choice functions $\left\{f^{\bar{K}, \bar{N}}: \mathcal{D}^{\bar{N}} \rightarrow 2^{\bar{K}} \backslash\{\emptyset\}\right\}_{\bar{K} \subseteq K, \bar{N} \subseteq N}$ strategy-proof on the cohesive dichotomous domain, consistent in individuals and neutral, then the derived family of social welfare functions $\left\{F_{f}^{\bar{N}}: \mathcal{D}^{\bar{N}} \rightarrow \mathcal{R}\right\}_{\bar{N} \subseteq N}$ is monotone and satisfies IIA, and (2) for all $\bar{N} \subseteq N$, if the social welfare function $F^{\bar{N}}: \mathcal{D}^{\bar{N}} \rightarrow \mathcal{R}$ is monotone and satisfies IIA, then the derived family of social choice functions $\left\{f_{F}^{\bar{K}, \bar{N}}: \mathcal{D}^{\bar{N}} \rightarrow 2^{\bar{K}} \backslash\{\emptyset\}\right\}_{\bar{K} \subseteq K}$ is strategy-proof on the cohesive dichotomous domain.

Proof: (1) Suppose that for some set of individuals $\bar{N} \subseteq N$, the derived social welfare function $F_{f}^{\bar{N}}: \mathcal{D}^{\bar{N}} \rightarrow \mathcal{R}$ does not satisfy IIA. Then, there are two preference profiles $D_{\bar{N}}, D_{\bar{N}}^{\prime} \in \mathcal{D}^{\bar{N}}$ which are such that for some pair of alternatives $(x, y), N\left(D_{\bar{N}} ; x, y\right)=N\left(D_{\bar{N}}^{\prime} ; x, y\right), N\left(D_{\bar{N}} ; y, x\right)=N\left(D_{\bar{N}}^{\prime} ; y, x\right)$, whereas the derived social orderings satisfy the conditions $x P_{f}\left(D_{\bar{N}}\right) y$ and $y R_{f}\left(D_{\bar{N}}^{\prime}\right) x$. By definition of the derived social welfare function $F_{f}^{\bar{N}}, f^{\{x, y\}}\left(D_{\bar{N}}\right)=\{x\}$ and $f^{\{x, y\}}\left(D_{\bar{N}}^{\prime}\right) \in\{\{y\},\{x, y\}\}$. Let $i \in C \subseteq \bar{N}$ if and only if $x P_{i} y$ or $y P_{i} x$. If $C=\emptyset$, then for all $i \in \bar{N}, x I_{i} y$. In this case $f^{\{x, y\}}\left(D_{\bar{N}}\right)=\{x, y\}$, because the family $\left\{f^{\bar{K}, \bar{N}}\right\}_{\bar{K} \subseteq K, \bar{N} \subseteq N}$ is neutral by assumption and the empty set cannot be selected. This is a contradiction to $f^{\{x, y\}}\left(D_{\bar{N}}\right)=\{x\}$, and therefore, $C \neq \emptyset$. Since $f^{\{x, y\}}\left(D_{\bar{N}}\right)=\{x\}$ by assumption, consistency in individuals implies that $f^{\{x, y\}}\left(\left.D_{\bar{N}}\right|_{C}\right)=\{x\}$. For simplicity let the preference profile $D_{C} \in \mathcal{D}^{C}$ be such that $D_{C}=\left.D_{\bar{N}}\right|_{C}$.

We prove in the next step that if $j \in C$, then $f^{\{x, y\}}\left(D_{j}^{\prime}, D_{C \backslash\{j\}}\right)=\{x\}$. Suppose otherwise, that is $f^{\{x, y\}}\left(D_{j}^{\prime}, D_{C \backslash\{j\}}\right) \in\{\{y\},\{x, y\}\}$. If $x P_{i} y$, then $j$ can manipulate $f^{\{x, y\}, C}$ at $\left(D_{j}^{\prime}, D_{C \backslash\{j\}}\right) \in \mathcal{D}^{C}$ via $D_{j} \in \mathcal{D}$. On the other hand, if $y P_{i} x$, then $j$ can manipulate $f^{\{x, y\}, C}$ at $D_{C} \in \mathcal{D}^{C}$ via $D_{j}^{\prime} \in \mathcal{D}$. This is a 
contradiction, and therefore, we can conclude that $f^{\{x, y\}}\left(D_{j}^{\prime}, D_{C \backslash\{j\}}\right)=\{x\}$.

Let $M \subset C$ be such that $j \in M$ and $2 \leq|M|<|C|$. Suppose that for all $\hat{M} \subseteq M$ which are such that $j \in \hat{M}, f^{\{x, y\}}\left(D_{\hat{M}}^{\prime}, D_{C \backslash \hat{M}}\right)=\{x\}$. We show that for all $\tilde{M}=M \cup\{i\}, i \in C \backslash M, f^{\{x, y\}}\left(D_{\tilde{M}}^{\prime}, D_{C \backslash \tilde{M}}\right)=\{x\}$. Suppose otherwise, that is $f^{\{x, y\}}\left(D_{\tilde{M}}^{\prime}, D_{C \backslash \tilde{M}}\right) \in\{\{y\},\{x, y\}\}$. If $x P_{i} y$, then $i$ can manipulate $f^{\{x, y\}, C}$ at $\left(D_{\tilde{M}}^{\prime}, D_{C \backslash \tilde{M}}\right) \in \mathcal{D}^{C}$ via $D_{i} \in \mathcal{D}$. On the other hand, if $y P_{i} x$, then $i$ can manipulate $f^{\{x, y\}, C}$ at $\left(D_{M}, D_{C \backslash M}\right) \in \mathcal{D}^{C}$ via $D_{i}^{\prime} \in \mathcal{D}^{C}$. Hence, it has to be that $f^{\{x, y\}}\left(D_{\tilde{M}}^{\prime}, D_{C \backslash \tilde{M}}\right)=\{x\}$. In particular, if $M=C \backslash\{i\}$, then $f^{\{x, y\}}\left(D_{\tilde{M}}^{\prime}, D_{C \backslash \tilde{M}}\right)=f^{\{x, y\}}\left(D_{C}^{\prime}\right)=\{x\}$. Finally, $f^{\{x, y\}}\left(D_{C}^{\prime}, D_{\bar{N} \backslash C}^{\prime}\right)=$ $f^{\{x, y\}}\left(D_{\bar{N}}^{\prime}\right)=\{x\}$, because the family $\left\{f^{\bar{K}, \bar{N}}\right\}_{\bar{K} \subseteq K, \bar{N} \subseteq N}$ is consistent in individuals and $x I_{i}^{\prime} y$ for all $i \in \bar{N} \backslash C$ (to see this remember that the preference profiles $D_{\bar{N}}, D_{\bar{N}}^{\prime} \in \mathcal{D}^{\bar{N}}$ satisfy the conditions $N\left(D_{\bar{N}} ; x, y\right)=N\left(D_{\bar{N}}^{\prime} ; x, y\right)$ and $N\left(D_{\bar{N}} ; y, x\right)=N\left(D_{\bar{N}}^{\prime} ; y, x\right)$; thus, $\left.x I_{i} y \Leftrightarrow x I_{i}^{\prime} y\right)$. This is a contradiction.

Suppose that for some set of individuals $\bar{N} \subseteq N$, the derived social welfare function $F_{f}^{\bar{N}}: \mathcal{D}^{\bar{N}} \rightarrow \mathcal{R}$ is not monotone. Then, there are two preference profiles $D_{\bar{N}}, D_{\bar{N}}^{\prime} \in \mathcal{D}^{\bar{N}}$ which are such that for some $i$ and some pair of alternatives $(x, y), i \notin N\left(D_{\bar{N}} ; x, y\right), i \in N\left(D_{\bar{N}}^{\prime} ; x, y\right)$ and $D_{\bar{N} \backslash\{i\}}^{\prime}=D_{\bar{N} \backslash\{i\}}$, whereas the derived social orderings satisfy the conditions $x R_{f}\left(D_{\bar{N}}\right) y$ and $y P_{f}\left(D_{\bar{N}}^{\prime}\right) x$. Observe that $i \notin N\left(D_{\bar{N}} ; x, y\right)$ is equivalent to $y \in G\left(D_{i}\right)$ and $x \in B\left(D_{i}\right)$. By definition of the derived social welfare function $F_{f}^{\bar{N}}, f^{\{x, y\}}\left(D_{\bar{N}}\right) \in\{\{x\},\{x, y\}\}$ and $f^{\{x, y\}}\left(D_{i}^{\prime}, D_{\bar{N} \backslash\{i\}}\right)=\{y\}$. Thus, $i$ can manipulate $f^{\{x, y\}, \bar{N}}$ at $D_{\bar{N}} \in \mathcal{D}^{\bar{N}}$ via $D_{i}^{\prime} \in \mathcal{D}$. This is a contradiction.

(2) Suppose that for some set of individuals $\bar{N} \subseteq N$, the derived family of social choice functions $\left\{f_{F}^{\bar{K}, \bar{N}}: \mathcal{D}^{\bar{N}} \rightarrow 2^{\bar{K}} \backslash\{\emptyset\}\right\}_{\bar{K} \subset K}$ is not strategy-proof on the cohesive dichotomous domain. Then, for for some $\bar{K} \subseteq K$ and $\bar{N} \subseteq N$, there is an $i \in \bar{N}$ who manipulates $f_{F}^{\bar{K}, \bar{N}}$ at $D_{\bar{N}} \in \mathcal{D}^{\bar{N}}$ via $D_{i}^{\prime} \in \mathcal{D}$, or $f_{F}^{\bar{K}}\left(D_{i}^{\prime}, D_{\bar{N} \backslash\{i\}}\right) \succ_{D_{i}} f_{F}^{\bar{K}}\left(D_{\bar{N}}\right)$. Define $S=f_{F}^{\bar{K}}\left(D_{\bar{N}}\right)$ and $T=f_{F}^{\bar{K}}\left(D_{i}^{\prime}, D_{\bar{N} \backslash\{i\}}\right)$. By definition of the cohesive preference relation $\succsim_{D_{i}}, f_{F}^{\bar{K}}\left(D_{i}^{\prime}, D_{\bar{N} \backslash\{i\}}\right) \succ_{D_{i}}$ $f_{F}^{\bar{K}}\left(D_{\bar{N}}\right)$ is equivalent to $\frac{\left|G\left(D_{i}\right) \cap T\right|}{|T|}>\frac{\left|G\left(D_{i}\right) \cap S\right|}{|S|}$. Observe that if the sets $S \backslash T$ (the alternatives that are removed from the image when moving from $S$ to $T$ ) and $T \backslash S$ (the alternatives that are added to the image when moving from $S$ 
to $T)$ are such that $S \backslash T \subseteq G\left(D_{i}\right)$ and $T \backslash S \subseteq B\left(D_{i}\right)$, then $f^{\bar{K}, \bar{N}}$ is not manipulable by $i$ at $D_{\bar{N}} \in \mathcal{D}^{\bar{N}}$ via $D_{i}^{\prime}$. Hence, either there is $x \in S \backslash T$ such that $x \in B\left(D_{i}\right)$ or there is $y \in T \backslash S$ such that $y \in G\left(D_{i}\right)$.

Case (a): Suppose there is a $x \in B\left(D_{i}\right)$ such that $x \in S \backslash T$. There are two cases to consider: (i) $T \backslash S \subseteq B\left(D_{i}\right)$ and (ii) for some $y \in T \backslash S, y \in G\left(D_{i}\right)$.

Case (a.i): Suppose that $T \backslash S \subseteq B\left(D_{i}\right)$. If $S \cap T \subseteq B\left(D_{i}\right)$, then $T \subseteq B\left(D_{i}\right)$ and $i$ cannot manipulate $f_{F}^{\bar{K}, \bar{N}}$ at $D \in \mathcal{D}^{\bar{N}}$ via $D_{i}^{\prime}$ by definition of the cohesive preferences extension. Hence, there is a $y \in S \cap T$ such that $y \in G\left(D_{i}\right)$. Since $x \in B\left(D_{i}\right)$ by assumption and $y \in G\left(D_{i}\right)$ by construction, $i \notin N\left(D_{\bar{N}} ; x, y\right)$ and $i \in N\left(D_{\bar{N}} ; y, x\right)$. Now apply the definition of the derived social welfare function to see that $x \in S \backslash T$ and $y \in T \cap S$ is equivalent to $x I\left(D_{\bar{N}}\right) y$ and $y P\left(D_{i}^{\prime}, D_{\bar{N} \backslash\{i\}}\right) x$, respectively. Notice that the social ordering restricted to $x$ and $y$ at $D_{\bar{N}} \in \mathcal{D}^{\bar{N}}$ depends only on the sets $N\left(D_{\bar{N}} ; x, y\right)$ and $N\left(D_{\bar{N}} ; y, x\right)$, because $F^{\bar{N}}$ satisfies IIA by assumption. Since $D_{j}^{\prime}=D_{j}$ for all $j \neq i$ and the social ordering restricted to $x$ and $y$ is different at $D_{\bar{N}} \in \mathcal{D}^{\bar{N}}$ and $D_{\bar{N}}^{\prime} \in \mathcal{D}^{\bar{N}}$, either $i \in N\left(\left(D_{i}^{\prime}, D_{\bar{N} \backslash\{i\}}\right) ; x, y\right)$ or $i \notin N\left(\left(D_{i}^{\prime}, D_{\bar{N} \backslash\{i\}}\right) ; y, x\right)$. Suppose that $i \notin N\left(\left(D_{i}^{\prime}, D_{\bar{N} \backslash\{i\}}\right) ; y, x\right)$. Then, it follows that $i \in N\left(\left(D_{i}^{\prime}, D_{\bar{N} \backslash\{i\}}\right) ; x, y\right)$, because the individuals have complete preferences on the set of alternatives. This together with $i \notin N\left(D_{\bar{N}} ; x, y\right)$, $D_{\bar{N} \backslash\{i\}}^{\prime}=D_{\bar{N} \backslash\{i\}}$ and $x I\left(D_{\bar{N}}\right) y$ implies that $x R\left(D_{i}^{\prime}, D_{\bar{N} \backslash\{i\}}\right) y$ by monotonicity of $F^{\bar{N}}$. This is a contradiction to $y P\left(D_{i}^{\prime}, D_{\bar{N} \backslash\{i\}}\right) x$.

Case (a.ii): If there is a $y \in T \backslash S$ such that $y \in G\left(D_{i}\right)$, then $x P\left(D_{\bar{N}}\right) y$ and $y P\left(D_{i}^{\prime}, D_{\bar{N} \backslash\{i\}}\right) x$ by definition of the derived family of social choice functions $\left\{f^{\bar{K}, \bar{N}}\right\}_{\bar{K} \subseteq K, \bar{N} \subseteq N}$. Now, it is possible to apply the same kind of argument as before.

Case (b): The construction of the proof of the second case is symmetric to the one of the first case. Therefore, it is omitted. 


\section{Three Characterizations of Approval Voting}

Although Proposition 1 is important on its own (we will use it later on derive a corollary), we use it mainly as an intermediate step in the first characterization of Approval Voting. To prove the main result of the paper we introduce a Lemma which states that if a family of social choice functions is consistent in alternatives, then the derived social preference relation is transitive and contains exactly the same information as the corresponding family of social choice functions. Hence, it is possible to go back and forth between social choice functions and social welfare functions.

Lemma 1 Assume that the family $\left\{f^{\bar{K}, \bar{N}}: \mathcal{D}^{\bar{N}} \rightarrow 2^{\bar{K}} \backslash\{\emptyset\}\right\}_{\bar{K} \subseteq K, \bar{N} \subseteq N}$ is consistent in alternatives. Then, (1) for all sets $\bar{N} \subseteq N$ and all preference profiles $D_{\bar{N}} \in \mathcal{D}^{\bar{N}}, R_{f}\left(D_{\bar{N}}\right)$ is transitive, and (2) for all sets $\bar{K} \subseteq K$ and $\bar{N} \subseteq N$, and all preference profiles $D_{\bar{N}} \in \mathcal{D}^{\bar{N}},\left.R_{f}^{1}\left(D_{\bar{N}}\right)\right|_{\bar{K}}=f^{\bar{K}}\left(D_{\bar{N}}\right)$.

Proof: See the Appendix.

If we restrict the analysis to social choice rules and add the properties of anonymity and strict monotonicity to the ones of neutrality and strategyproofness on the cohesive dichotomous domain, then we are able to characterize Approval Voting with the help of Proposition 1 and Lemma 1.

Theorem 1 The social choice rule $\left\{f^{\bar{K}, \bar{N}}: \mathcal{D}^{\bar{N}} \rightarrow 2^{\bar{K}} \backslash\{\emptyset\}\right\}_{\bar{K} \subseteq K, \bar{N} \subseteq N}$ is neutral, anonymous, strictly monotone and strategy-proof on the cohesive dichotomous domain if and only if $\left\{f^{\bar{K}, \bar{N}}\right\}_{\bar{K} \subseteq K, \bar{N} \subseteq N}$ is Approval Voting.

Proof: It is easy to see that the social choice rule corresponding to Approval Voting is neutral, anonymous, strictly monotone and strategy-proof on the cohesive dichotomous domain. To prove the other implication suppose that the social choice rule $\left\{f^{\bar{K}, \bar{N}}: \mathcal{D}^{\bar{N}} \rightarrow 2^{\bar{K}} \backslash\{\emptyset\}\right\}_{\bar{K} \subseteq K, \bar{N} \subseteq N}$ satisfies the four properties. It remains to prove that given the sets $\bar{K} \subseteq K$ and $\bar{N} \subseteq N$, and the preference profile $D_{\bar{N}} \in \mathcal{D}^{\bar{N}}, f^{\bar{K}}\left(D_{\bar{N}}\right)=\left\{x \in \bar{K}: N_{x}\left(D_{\bar{N}}\right) \geq N_{y}\left(D_{\bar{N}}\right)\right.$ for all $\left.y \in \bar{K}\right\}$.

In an intermediate step it is shown that the derived social welfare function orders the alternatives according to Approval Voting, that is for all sets of in- 
dividuals $\bar{N} \subseteq N$, all preference profiles $D_{\bar{N}} \in \mathcal{D}^{\bar{N}}$ and all pairs of alternatives $(x, y),\left(\right.$ a) if $N_{x}\left(D_{\bar{N}}\right)=N_{y}\left(D_{\bar{N}}\right)$, then $x I_{f}\left(D_{\bar{N}}\right) y$ and (b) if $N_{x}\left(D_{\bar{N}}\right)>N_{y}\left(D_{\bar{N}}\right)$, then $x P_{f}\left(D_{\bar{N}}\right) y$. By the first part of Proposition 1, the derived family of social welfare functions $\left\{F_{f}^{\bar{N}}: \mathcal{D}^{\bar{N}} \rightarrow \mathcal{R}\right\}_{\bar{N} \subseteq N}$ satisfies IIA. Hence, for all sets of individuals $\bar{N} \subseteq N$, all preference profiles $D_{\bar{N}} \in \mathcal{D}^{\bar{N}}$ and all pairs of alternatives $(x, y),\left.R\left(D_{\bar{N}}\right)\right|_{\{x, y\}}$ is a function of the sets $N\left(D_{\bar{N}} ; x, y\right)$ and $N\left(D_{\bar{N}} ; y, x\right)$. Moreover, if the social choice rule $\left\{f^{\bar{K}, \bar{N}}\right\}_{\bar{K} \subseteq K, \bar{N} \subseteq N}$ is anonymous, then the derived family of social welfare function $\left\{F_{f}^{\bar{N}}\right\}_{\bar{N} \subseteq N}$ is anonymous, too. Both results together imply that given a set of individuals $\bar{N} \subseteq N$, a preference profile $D_{\bar{N}} \in$ $\mathcal{D}^{\bar{N}}$, and a pair of alternatives $(x, y),\left.R_{f}\left(D_{\bar{N}}\right)\right|_{\{x, y\}}$ is a function of the numbers $\left|N\left(D_{\bar{N}} ; x, y\right)\right|$ and $\left|N\left(D_{\bar{N}} ; y, x\right)\right|$. Observe that the former numbers are equal to $N_{x}\left(D_{\bar{N}}\right)+\left|\left\{i \in \bar{N}: x, y \in B\left(D_{i}\right)\right\}\right|$ and $N_{y}\left(D_{\bar{N}}\right)+\left|\left\{i \in \bar{N}: x, y \in B\left(D_{i}\right)\right\}\right|$.

(a) Suppose that given a set $\bar{N} \subseteq N$, a preference profile $D_{\bar{N}} \in \mathcal{D}^{\bar{N}}$, and a pair of alternatives $(x, y), N_{x}\left(D_{\bar{N}}\right)=N_{y}\left(D_{\bar{N}}\right)$, but the derived social welfare function $F_{f}^{\bar{N}}$ is such that $x P\left(D_{\bar{N}}\right) y$. Apply the definition of the derived social welfare function $F_{f}^{\bar{N}}$ to see that $f^{\{x, y\}}\left(D_{\bar{N}}\right)=\{x\}$. Next, let the permutation $\mu$ of $K$ satisfy $\mu(x)=y$ and $\mu(y)=x$. Neutrality implies that $f^{\{x, y\}}\left(\mu\left(D_{\bar{N}}\right)\right)=$ $\mu\left(f^{\{x, y\}}\left(D_{\bar{N}}\right)\right)=\{y\}$, and therefore, $y P_{f}\left(\mu\left(D_{\bar{N}}\right)\right) x$ by the definition of the derived social welfare function $F_{f}^{\bar{N}}$. The assumption $N_{x}\left(D_{\bar{N}}\right)=N_{y}\left(D_{\bar{N}}\right)$ implies that $\left|N\left(D_{\bar{N}} ; x, y\right)\right|=\left|N\left(D_{\bar{N}} ; y, x\right)\right|$ and due to the construction of the preference profile $D_{\bar{N}} \in \mathcal{D}^{\bar{N}}$ we deduce that $\left|N\left(\mu\left(D_{\bar{N}}\right) ; x, y\right)\right|=\left|N\left(D_{\bar{N}} ; x, y\right)\right|$ and $\left|N\left(\mu\left(D_{\bar{N}}\right) ; y, x\right)\right|=\left|N\left(D_{\bar{N}} ; y, x\right)\right|$. Since the social ordering between $x$ and $y$ just depends on these numbers, the condition $\left.F_{f}\left(\mu\left(D_{\bar{N}}\right)\right)\right|_{\{x, y\}}=\left.F_{f}\left(D_{\bar{N}}\right)\right|_{\{x, y\}}$ has to be satisfied. We have reached a contradiction, because $y P_{f}\left(\mu\left(D_{\bar{N}}\right)\right) x$ and $x P\left(D_{\bar{N}}\right) y$. Therefore, it has to be that $x I_{f}\left(D_{\bar{N}}\right) y$.

(b) Suppose that given a set $\bar{N} \subseteq N$, a preference profile $D_{\bar{N}} \in \mathcal{D}^{\bar{N}}$, and a pair of alternatives $(x, y), N_{x}\left(D_{\bar{N}}\right)-N_{y}\left(D_{\bar{N}}\right)=1$. Construct the preference profile $D_{\bar{N}}^{\prime} \in \mathcal{D}^{\bar{N}}$ in the following way: For some individual $i$ whose preference relation $D_{i}$ is such that $x \in G\left(D_{i}\right)$ and $y \in B\left(D_{i}\right)$, the preference relation $D_{i}^{\prime}$ satisfies the condition $G\left(D_{i}^{\prime}\right)=G\left(D_{i}\right) \backslash\{x\}$. Moreover, let $D_{j}^{\prime}=D_{j}$ for all $j \neq i$. Since at the preference profile $D_{\bar{N}}^{\prime} \in \mathcal{D}^{\bar{N}}, N_{x}\left(D_{\bar{N}}^{\prime}\right)=N_{y}\left(D_{\bar{N}}^{\prime}\right)$, it follows from 
the former part of the proof that $x I_{f}\left(D_{\bar{N}}^{\prime}\right) y$ or $f^{\{x, y\}}\left(D_{\bar{N}}^{\prime}\right)=\{x, y\}$. Finally, apply the definition of strict monotonicity to see that $f^{\{x, y\}}\left(D_{\bar{N}}\right)=\{x\}$ which is equivalent to $x P\left(D_{\bar{N}}\right) y$.

Let $2 \leq m<\bar{n}$. Suppose that for all $\bar{m} \leq m$, if, $N_{x}\left(D_{\bar{N}}\right)-N_{y}\left(D_{\bar{N}}\right)=\bar{m}$, then $x P_{f}\left(D_{\bar{N}}\right) y$. We show that if $N_{x}\left(D_{\bar{N}}\right)-N_{y}\left(D_{\bar{N}}\right)=\bar{m}+1$, then $x P_{f}\left(D_{\bar{N}}\right) y$. Construct the preference profile $D_{\bar{N}}^{\prime} \in \mathcal{D}^{\bar{N}}$ in the following way: For some individual $i$ whose preference relation $D_{i}$ is such that $x \in G\left(D_{i}\right)$ and $y \in$ $B\left(D_{i}\right)$, the preference relation $D_{i}^{\prime}$ satisfies the condition $G\left(D_{i}^{\prime}\right)=G\left(D_{i}\right) \backslash\{x\}$. Moreover, let $D_{j}^{\prime}=D_{j}$ for all $j \neq i$. Since at the preference profile $D_{\bar{N}}^{\prime} \in \mathcal{D}^{\bar{N}}$, $N_{x}\left(D_{\bar{N}}^{\prime}\right)-N_{y}\left(D_{\bar{N}}^{\prime}\right)=\bar{m}$, it has to be that $x P_{f}\left(D_{\bar{N}}^{\prime}\right) y$ by assumption. The former condition is equivalent to $f^{\{x, y\}}\left(D_{\bar{N}}^{\prime}\right)=\{x\}$. Finally, apply the definition of strict monotonicity to see that $f^{\{x, y\}}\left(D_{\bar{N}}\right)=\{x\}$ which is equivalent to $x P\left(D_{\bar{N}}\right) y$.

So far, we have shown that for all sets of feasible alternatives $\bar{K} \subseteq K$, all sets of individuals $\bar{N} \subseteq N$ and all the preference profiles $D_{\bar{N}} \in \mathcal{D}^{\bar{N}}$, the derived social welfare function $R_{f}\left(D_{\bar{N}}\right)$ satisfies $\left.R_{f}^{1}\left(D_{\bar{N}}\right)\right|_{\bar{K}}=\left\{x \in \bar{K}: N_{x}\left(D_{\bar{N}}\right) \geq\right.$ $N_{y}\left(D_{\bar{N}}\right)$ for all $\left.y \in \bar{K}\right\}$. Since for all sets $\bar{K} \subseteq K$ and $\bar{N} \subseteq N$, and all preference profiles $D_{\bar{N}} \in \mathcal{D}^{\bar{N}}, f_{F_{f}}^{\bar{K}}\left(D_{\bar{N}}\right)=\left.R_{f}^{1}\left(D_{\bar{N}}\right)\right|_{\bar{K}}$ by definition of the derived social social function and $\left.R_{f}^{1}\left(D_{\bar{N}}\right)\right|_{\bar{K}}=f^{\bar{K}}\left(D_{\bar{N}}\right)$ by Lemma 1, we deduce finally that $f^{\bar{K}}\left(D_{\bar{N}}\right)=\left\{x \in \bar{K}: N_{x}\left(D_{\bar{N}}\right) \geq N_{y}\left(D_{\bar{N}}\right)\right.$ for all $\left.y \in \bar{K}\right\}$.

In the Appendix it is shown that Theorem 1 is tight. The result which is closest to Theorem 1 is due to Fishburn [8] who characterizes Approval Voting as the only anonymous family of social choice functions (he allows only the set of individuals to vary and considers the set of feasible alternatives to be fixed) that is strategy-proofness, neutral and consistent. ${ }^{7}$ Using the current notation Fishburn's consistency property is defined as follows: Let $\overline{\mathcal{D}}$ be the domain of

\footnotetext{
${ }^{7}$ Actually Fishburn [8] uses the following extension to preferences on subsets of alternatives: The weak preference relation $\succsim_{D_{i}}$ on $2^{K} \backslash\{\emptyset\}$ is as follows: (a) $\{x\} \dot{\succ}_{R_{i}}\{x, y\} \dot{\succ}_{R_{i}}\{y\}$ if $x \in$ $G\left(R_{i}\right)$ and $y \in B\left(R_{i}\right)$; (b) for all $S, T \in 2^{K} \backslash\{\emptyset\}, S \dot{\sim}_{R_{i}} T$ if $T \subseteq B\left(R_{i}\right)$ or $S \subseteq G\left(R_{i}\right)$ or $\left[S \backslash T \subseteq G\left(R_{i}\right)\right.$ and $\left.T \backslash S \subseteq B\left(R_{i}\right)\right]$. This preference extension is weaker than the cohesive preferences extension proposed in this paper, but its disadvantage is that it induces a non complete ordering on $2^{K} \backslash\{\emptyset\}$. Consequently, one has to check for possible manipulations only for all comparable non-empty subsets of $K$.
} 
dichotomous preferences without the two preference relations indicating that an individual is indifferent between all alternatives. The anonymous family of social choice functions $\left\{f: \overline{\mathcal{D}}^{\bar{N}} \rightarrow 2^{K} \backslash\{\emptyset\}\right\}_{\bar{N} \subseteq N}$ is said to be consistent if for all sets of individuals $\hat{N}, \tilde{N} \subseteq N, \hat{N} \cap \tilde{N}=\emptyset$, and all pairs of preference profiles $\bar{D}_{\hat{N}} \in \overline{\mathcal{D}}^{\hat{N}}$ and $\overline{\mathcal{D}}_{\tilde{N}} \in \overline{\mathcal{D}}^{\tilde{N}}$ which satisfy $f\left(\bar{D}_{\hat{N}}\right) \cap f\left(\bar{D}_{\tilde{N}}\right) \neq \emptyset$, the condition $f\left(\bar{D}_{\hat{N} \cup \tilde{N}}\right)=f\left(\bar{D}_{\hat{N}}\right) \cap f\left(\bar{D}_{\tilde{N}}\right)$ holds. ${ }^{8} \quad$ Since consistency in individuals has the same flavor as deleting the preference relations expressing indifference between all alternatives from the domain, there are three main differences between the two results: First, here we use the property of strict monotonicity instead of consistency (notice that both properties are independent from each other). Second, Fishburn [8] studies anonymous social choice function, whereas in this paper we do assume anonymity straight from the beginning. Finally, our analysis is more general, because we allow the set of feasible alternatives to vary as well.

To see why we have implicitly also characterized the social welfare function corresponding to Approval Voting it is only necessary to consider Theorem 1 together with Proposition 1. Fix $\bar{N}=N$ in the second part of Proposition 1. Hence, if the strictly monotone social welfare function $F$ satisfies IIA, then the derived family of social choice functions $\left\{f_{F}^{\bar{K}}\right\}_{\bar{K} \subseteq K}$ is strategy-proof on the cohesive dichotomous domain. Since the properties of neutrality, anonymity and strict monotonicity translate from social welfare functions to social choice functions, the derived family of social choice functions is consistent in alternatives by construction and the set of voters is fixed, we can apply Theorem 1 in order deduce that $\left\{f_{F}^{\bar{K}}\right\}_{\bar{K} \subseteq K}$ is equal to Approval Voting. Finally, just apply the definition of the derived social welfare function to obtain the following result.

Corollary 1 The social welfare functions $F: \mathcal{D}^{N} \rightarrow \mathcal{R}$ is strictly monotone, neutral, anonymous and satisfies IIA if and only if for all preference profiles $D \in \mathcal{D}^{\bar{N}}$ and for all pairs of alternatives $(x, y) \in K^{2}, x R(D) y$ if and only if $N_{x}(D) \geq N_{y}(D)$.

\footnotetext{
${ }^{8}$ The preference profile $\bar{D}_{\hat{N} \cup \tilde{N}} \in \mathcal{D}^{\hat{N} \cup \tilde{N}}$ is obtained by unifying the other two preference profiles, that is $\left.\bar{D}_{\hat{N} \cup \tilde{N}}\right|_{\hat{N}}=\bar{D}_{\hat{N}}$ and $\left.\bar{D}_{\hat{N} \cup \tilde{N}}\right|_{\tilde{N}}=\bar{D}_{\tilde{N}}$.
} 
This result can be found in Moulin [13] as an exercise and can be interpreted as an extension of May's Theorem [11] which reads as follows: Suppose that $K=2$. The social welfare function $F: \mathcal{R}^{N} \rightarrow \mathcal{R}$ is anonymous, neutral, and strictly monotone if and only if $F$ is the social welfare function associated to the Majority Rule, e.g. for all $R \in \mathcal{R}^{N}$ and all $(x, y) \in K^{2}, x F(R) y$ if and only if $\left|\left\{i \in N: x R_{i} y\right\}\right| \geq\left|\left\{i \in N: y R_{i} x\right\}\right|$. Since Approval Voting is equivalent to the Condorcet Rule on the dichotomous preferences domain according to Brams and Fishburn [5], Corollary 1 shows that May's Theorem can be extended to any number of alternatives if we add IIA to the original properties.

For the rest of the Section we restrict our attention to the special case when all individuals reveal their preferences and all alternatives are feasible. The idea behind the following two characterizations of Approval Voting is to identify in a first step a property such that for all preference profiles $D \in \mathcal{D}^{N}$ the social choice function $f$ is a function of the $K$-dimensional vector $\left(N_{x}(D)\right)_{x \in K}$. Formally, the social choice function $f: \mathcal{D}^{N} \rightarrow 2^{K} \backslash\{\emptyset\}$ depends on the support of the alternatives if for all pairs of preference profiles $D, D^{\prime} \in \mathcal{D}^{N}$ which are such that $N_{x}(D)=N_{x}\left(D^{\prime}\right)$ for all $x \in K$, the condition $f(D)=f\left(D^{\prime}\right)$ holds. This property is stronger than the one obtained in the proof of Theorem 1 , because there strategy-proofness, neutrality and anonymity assure that for all sets $\bar{K} \subseteq K$ and $\bar{N} \subseteq N$, and all preference profiles $D_{\bar{N}} \in \mathcal{D}^{\bar{N}}$, the social choice rule $\left\{f^{\bar{K}, \bar{N}}\right\}_{\bar{K} \subseteq K, \bar{N} \subseteq N}$ is a function of the $\frac{\bar{K}(\bar{K}+1)}{2}$-dimensional vector $\left(\left(N_{x}\left(D_{\bar{N}}\right)\right)_{x \in \bar{K}},\left(\left|i \in \bar{N}: x, y \in B\left(D_{i}\right)\right|\right)_{x, y \in \bar{K}}\right)$. Next, we introduce the necessary definitions. The preference profiles $D, D^{\prime} \in \mathcal{D}^{N}$ are said to be $x$-symmetric if for some alternative $x$ and some pair of individuals $(i, j), G\left(D_{i}^{\prime}\right) \cup\{x\}=G\left(D_{i}\right)$, $G\left(D_{j}^{\prime}\right)=G\left(D_{j}\right) \cup\{x\}$, where $x \notin G\left(D_{i}^{\prime}\right) \cup G\left(D_{j}\right)$, and $D_{l}^{\prime}=D_{l}$ for all $l \neq i, j$.

Definition 7 The social choice function $f: \mathcal{D}^{N} \rightarrow 2^{K} \backslash\{\emptyset\}$ is said to be strictly symmetric if for all $x$-symmetric preference profiles $D, D^{\prime} \in \mathcal{D}^{N}, f(D)=f\left(D^{\prime}\right)$.

Strict symmetry is a rather strong independence condition stating that, given $D_{i} \in \mathcal{D}$, the effect on the social choice function $f$ of alternative $x$ being good neither depends on the name of the individual (anonymity) nor on the set 
of good alternatives $G\left(D_{i}\right)$.

Lemma 2 The social choice function $f: \mathcal{D}^{N} \rightarrow 2^{K} \backslash\{\emptyset\}$ is strictly symmetric if and only if $f$ depends on the support of the alternatives.

Proof: See the Appendix.

We show next that if we add the property of neutrality to the one of strict symmetry and two alternatives receive the same support at a particular preference profile, then either both alternatives or none of them is selected. Formally, the social choice function $f: \mathcal{D}^{N} \rightarrow 2^{K} \backslash\{\emptyset\}$ treats alternatives with the same support similarly if for all preference profiles $D \in \mathcal{D}^{N}$ which are such that $N_{x}(D)=N_{y}(D)$ for some pair of alternatives $(x, y), x \in f(D)$ if and only if $y \in f(D)$.

Lemma 3 If the social choice function $f: \mathcal{D}^{N} \rightarrow 2^{K} \backslash\{\emptyset\}$ is strictly symmetric and neutral, Then $f$ treats alternatives with the same support similarly.

Proof: See the Appendix.

Finally, we include the property of strict monotonicity in order to finish the second characterization of Approval Voting.

Theorem 2 The social choice function $f: \mathcal{D}^{N} \rightarrow 2^{K} \backslash\{\emptyset\}$ is strictly symmetric, neutral, and strictly monotone if and only if $f$ is Approval Voting.

Proof: It is easy to see that Approval Voting is strictly symmetric, neutral, and strictly monotonic. To prove the other inclusion suppose that the social choice function $f: \mathcal{D}^{N} \rightarrow 2^{K} \backslash\{\emptyset\}$ satisfies the three properties. It remains to establish that for all preference profiles $D^{\prime \prime} \in \mathcal{D}^{N}, x \in f\left(D^{\prime \prime}\right)$ if and only if $N_{x}\left(D^{\prime \prime}\right) \geq N_{y}\left(D^{\prime \prime}\right)$ for all $y \in K$.

Consider the preference profile $D^{\prime} \in \mathcal{D}^{N}$ which is such that for all $x \in K$ and $i \in N, x \in G\left(D_{i}^{\prime}\right)$ if and only if $i \leq N_{x}\left(D^{\prime \prime}\right)$. Since $N_{y}\left(D^{\prime}\right)=N_{y}\left(D^{\prime \prime}\right)$ for all $y \in K$ and $f$ depends on the support of the alternatives by Lemma 2, $f\left(D^{\prime}\right)=f\left(D^{\prime \prime}\right)$. Define $p=\min _{y \in K} N_{y}\left(D^{\prime}\right)$, and $q=\max _{y \in K} N_{y}\left(D^{\prime}\right)$, respectively. Notice that $0 \leq p \leq q \leq n$. Moreover, the preference profile $D^{\prime} \in \mathcal{D}^{N}$ has 
the following three properties: (i) for all $i \leq p, G\left(D_{i}^{\prime}\right)=K$, (ii) for all $j>q$, $G\left(D_{j}^{\prime}\right)=\emptyset$, and (iii) for all $l, p \leq l \leq q, G\left(D_{l+1}^{\prime}\right) \subseteq G\left(D_{l}^{\prime}\right)$. Next, consider the preference profile $D \in \mathcal{D}^{N}$ which is such that for all $x \in K$ and $i \in N$, $x \in G\left(D_{i}\right)$ if and only if $i \leq p$. Then, $f(D)=K$, because $N_{y}(D)=p$ for all $y \in K$ and $f$ treats all alternatives with the same support similarly by Lemma 3. Next, verify the following three properties of the preference profile $D \in \mathcal{D}^{N}$ : (i) for all $i \in N, i \leq p, G\left(D_{i}\right)=K$, (ii) for all $j \in N, j>q, G\left(D_{j}\right)=\emptyset$, and (iii) for all $l \in N, p<l \leq q, G\left(D_{l}\right)=\emptyset$. Hence, only individual $l, p \leq l \leq q$, has different preferences according to the preference profiles $D \in \mathcal{D}^{N}$ and $D^{\prime} \in \mathcal{D}^{N}$.

Given $D^{p+1}=\left(D_{1}^{\prime}, \ldots, D_{p+1}^{\prime}, D_{p+2}, \ldots, D_{q}, D_{q+1}^{\prime}, \ldots D_{n}^{\prime}\right) \in \mathcal{D}^{N}$, we are going to prove that $f\left(D^{p+1}\right)=G\left(D_{p+1}^{\prime}\right)$. Since the preference profile $D^{p+1} \in \mathcal{D}^{N}$ satisfies the conditions $G\left(D_{p+1}^{p+1}\right)=G\left(D_{p+1}\right) \cup G\left(D_{p+1}^{\prime}\right), D_{-(p+1)}^{p+1}=D_{-(p+1)}$, and $G\left(D_{p+1}^{\prime}\right) \subseteq f(D)=K$, strict monotonicity of $f$ implies that $f\left(D^{p+1}\right)=$ $G\left(D_{p+1}^{\prime}\right)$. Let $p+2 \leq t<q$ and define $D^{t} \in \mathcal{D}^{N}$ for the generic integer $t$ as $D^{t}=\left(D_{1}^{\prime}, \ldots, D_{t}^{\prime}, D_{t+1}, \ldots, D_{q}, D_{q+1}^{\prime}, \ldots, D_{n}^{\prime}\right) \in \mathcal{D}^{N}$. Suppose that for all $2 \leq \bar{t} \leq t, f\left(D^{\bar{t}}\right)=G\left(D_{\bar{t}}^{\prime}\right)$. It remains to prove that $f\left(D^{t+1}\right)=G\left(D_{t+1}^{\prime}\right)$. Since the preference profile $D^{t+1} \in \mathcal{D}^{N}$ satisfies the conditions $G\left(D_{t+1}^{t+1}\right)=$ $G\left(D_{t+1}^{t}\right) \cup G\left(D_{t+1}^{\prime}\right), D_{-(t+1)}^{t+1}=D_{-(t+1)}^{t}$, and $G\left(D_{t+1}^{\prime}\right) \subseteq G\left(D_{t}^{\prime}\right)=f\left(D^{t}\right)$, strict monotonicity of $f$ implies that $f\left(D^{t+1}\right)=G\left(D_{t+1}^{\prime}\right)$. Therefore, it follows for the special when $q=t-1$ that, given $D^{q} \in \mathcal{D}^{N}, f\left(D^{q}\right)=G\left(D_{q}^{\prime}\right)$. Since the preference profile $D^{q} \in \mathcal{D}^{N}$ is equal to $D^{\prime} \in \mathcal{D}^{N}$ by construction, $f\left(D^{\prime}\right)=$ $G\left(D_{q}^{\prime}\right)$.

Finally, we are going to establish that $x \in f\left(D^{\prime \prime}\right)$ if and only if $N_{x}\left(D^{\prime \prime}\right) \geq$ $N_{y}\left(D^{\prime \prime}\right)$ for all $y \in K$. Suppose that $x \in f\left(D^{\prime \prime}\right)$. Then, $x \in G\left(D_{q}^{\prime}\right)$, because by construction of the preference profile $D^{\prime} \in \mathcal{D}^{N}, f\left(D^{\prime \prime}\right)=f\left(D^{\prime}\right)=G\left(D_{q}^{\prime}\right)$. Moreover, $N_{x}\left(D^{\prime}\right) \geq N_{y}\left(D^{\prime}\right)$ for all $y \in K$ as well by construction of the preference profile $D^{\prime} \in \mathcal{D}^{N}$. Finally, since $N_{z}\left(D^{\prime \prime}\right)=N_{z}\left(D^{\prime}\right)$ for all $z \in K$, the first inclusion has to be true. To show the other inclusion suppose that $N_{x}\left(D^{\prime \prime}\right) \geq$ $N_{y}\left(D^{\prime \prime}\right)$ for all $y \in K$ and consider the preference profile $D^{\prime} \in \mathcal{D}^{N}$ which is as described above. Since $N_{z}\left(D^{\prime \prime}\right)=N_{z}\left(D^{\prime}\right)$ for all $z \in K, N_{x}\left(D^{\prime}\right) \geq N_{y}\left(D^{\prime}\right)$ for all $y \in K$. Therefore, $x \in G\left(D_{q}^{\prime}\right)$ by construction of the preference profile 
$D^{\prime} \in \mathcal{D}^{N}$. The result follows, because $f\left(D^{\prime \prime}\right)=f\left(D^{\prime}\right)=G\left(D_{q}^{\prime}\right)$

The examples in the Appendix show that Theorem 2 is tight. Theorem 2 is inspired by the following version of May's Theorem [11]: Suppose that $K=2$. The social choice function $f: \mathcal{R}^{N} \rightarrow\{\{x\},\{x, y\},\{y\}\}$ is anonymous, neutral, and strictly monotone if and only if $f$ is the Majority Rule. Hence, Theorem 2 shows that May's Theorem can be extended to any number of alternatives if anonymity is replaced by the stronger property of strict symmetry. The last result of the paper states that under strict symmetry and neutrality, strict monotonicity can be replaced by the property of efficiency (there is no other non-empty subset of alternative such that some individual is better off and no individual is worse off according to her/his cohesive preferences).

Definition 8 The social choice function $f: \mathcal{D}^{N} \rightarrow 2^{K} \backslash\{\emptyset\}$ is said to be efficient if for all preference profiles $D \in \mathcal{D}^{N}$, there does not exist a set $S \subseteq K$ such that $S \succsim_{D_{i}} f(D)$ for all $i \in N$ and $S \succ_{D_{j}} f(D)$ for some $j \in N$.

Theorem 3 The social choice function $f: \mathcal{D}^{N} \rightarrow 2^{K} \backslash\{\emptyset\}$ is strictly symmetric, neutral, and efficient if and only if $f$ is Approval Voting.

Proof: It is straightforward to see that Approval Voting is strictly symmetric, neutral, and efficient. To prove the other implication suppose that the social choice function $f: \mathcal{D}^{N} \rightarrow 2^{K} \backslash\{\emptyset\}$ satisfies the three properties. It remains to establish that for all preference profiles $D^{\prime \prime} \in \mathcal{D}^{N}, x \in f\left(D^{\prime \prime}\right)$ if and only if $N_{x}\left(D^{\prime \prime}\right) \geq N_{y}\left(D^{\prime \prime}\right)$ for all $y \in K$.

Construct the preference profile $D^{\prime} \in \mathcal{D}^{N}$ in the same as in the proof of Theorem 2, that is $x \in G\left(D_{i}^{\prime}\right)$ if and only if $i \leq N_{x}\left(D^{\prime \prime}\right)$. Since $N_{y}\left(D^{\prime}\right)=N_{y}\left(D^{\prime \prime}\right)$ for all $y \in K$ and $f$ depends on the support of the alternatives by Lemma 2, $f\left(D^{\prime}\right)=f\left(D^{\prime \prime}\right)$. Define $p=\min _{y \in K} N_{y}\left(D^{\prime}\right)$ and $q=\max _{y \in K} N_{y}\left(D^{\prime}\right)$, respectively. Next, we prove that $f\left(D^{\prime}\right) \subseteq G\left(D_{q}^{\prime}\right)$ by efficiency. Suppose otherwise, that is for some $y \in K, y \in f\left(D^{\prime}\right)$ and $y \notin G\left(D_{q}^{\prime}\right)$. By the construction of the preference profile $D^{\prime} \in \mathcal{D}^{N}, p \leq m \equiv N_{y}\left(D^{\prime}\right)<q$. Let $x \in G\left(D_{q}^{\prime}\right)$ and observe that for all $i \in N,\{x\} \succsim_{D_{i}} f\left(D^{\prime}\right)$ due to the cohesive preference extension. Moreover, for individual $q,\{x\} \succ_{D_{q}} f\left(D^{\prime}\right)$, because $x \in G\left(D_{q}^{\prime}\right)$ and $y \notin G\left(D_{q}^{\prime}\right)$. We 
conclude that $f$ is not efficient, and therefore, $f\left(D^{\prime}\right) \subseteq G\left(D_{q}^{\prime}\right)$. Finally, notice that $f\left(D^{\prime}\right)=G\left(D_{q}^{\prime}\right)$, because for all $x \in G\left(D_{q}^{\prime}\right), N_{x}\left(D^{\prime}\right)=q$ and $f$ treats alternatives with the same support similarly according to Lemma 3. To finish the proof one can apply the same argument as in the proof of Theorem 2 to see that $x \in f\left(D^{\prime \prime}\right)$ if and only if $N_{x}\left(D^{\prime \prime}\right) \geq N_{y}\left(D^{\prime \prime}\right)$ for all $y \in K$.

In the Appendix we show that Theorem 3 is tight as well. Given the results of Theorem 2 and 3 the question of whether or not it is possible to apply in Theorem 1 efficiency instead of strict monotonicity arises. Consider the following example to see why this is not possible.

Example 3: Let $K=\{x, y\}$. For all sets of individuals $\bar{N} \subseteq N$ and all preference profiles $D_{\bar{N}} \in \mathcal{D}^{\bar{N}}$, (a) if there exists an alternative $x \in K$ such that $x R_{i} y$ for all $i \in \bar{N}$, then $g\left(D_{\bar{N}}\right)=\left\{x \in K: x R_{i} y\right.$ for all $\left.i \in \bar{N}\right\}$. Otherwise, $x \in$ $g\left(D_{\bar{N}}\right)$ if and only if $N_{x}(D)-N_{y}(D) \geq-1$. This modification of the qualitative majority rule is efficient, strategy-proof, neutral and anonymous but different from Approval Voting. To see this consider the preference profile $\left(D_{1}, D_{2}\right)$ which is such that $G\left(D_{1}\right)=\{x, y\}$ and $G\left(D_{2}\right)=\{x\}$. Then $f_{A}\left(D_{1}, D_{2}\right)=\{x\}$, whereas $g\left(D_{1}, D_{2}\right)=\{x, y\}$.

\section{References}

[1] S. Barberà, B. Dutta, and A. Sen, Strategy-proof Social Choice Correspondences, Journal of Economic Theory 101 (2001), 374-394.

[2] S. Barberà, H. Sonnenschein, and L. Zhou, Voting by Committees, Econometrica 59 (1991), 595-609.

[3] D. Blair and E. Muller, Esseential Aggregation Procedures on Restricted Domains of Preferences, Journal of Economic Theory 30 (1983), 34-53.

[4] S. Brams and P. Fishburn, Going from Theory to Practice: The Mixed Success of Approval Voting, forthcoming: Social Choice and Welfare. 
[5] _ Approval Voting, American Political Science Review 72 (1978), $831-847$.

[6] S. Ching and L. Zhou, Multi-valued Strategy-Proof Social Choice Rules, Social Choice and Welfare 19 (2002), 569-580.

[7] J. Duggan and T. Schwartz, Strategic Manipulability without Resoluteness or Shared Beliefs: Gibbard-Satterthwaite Generalized, Social Choice and Welfare 17 (2000), 85-93.

[8] P. Fishburn, Symmetric and Consistent Aggregation with Dichotomous Preferences, in "Aggregation and Revelation of Preferences" (ed. J. Laffont), North-Holland, Amsterdam, 1978.

[9] _ Axioms for Approval Voting: Direct Proof, Journal of Economic Theory 19 (1978), 180-185.

[10] T. Groves and M. Loeb, Incentives and Public Goods, Journal of Public Economics 43 (1975), 211-226.

[11] K. May, A Set of Independent Necessary and Sufficient Conditions for Simple Majority Decision, Econometrica 20 (1952), 680-684.

[12] H. Moulin, On Strategy-proofness and Single Peakedness, Public Choice 35 (1980), 437-455.

[13] _ Axioms of Cooperative Decision Making, Cambridge University Press, 1988.

[14] A. Sen and P. Pattanaik, Necessary and Sufficient Conditions for Rational Choice under Majority Decision, Journal of Economic Theory 1 (1969), 178-202.

[15] Y. Sprumont, The Division Problem with Single-Peaked Preferences: A Characterization of the Uniform Allocation Rule, Econometrica 39 (1991), $672-684$.

[16] M. Vorsatz, Scoring Rules on Dichotomous Preferences, Mimeo (2004). 


\section{Appendix}

\section{Proofs of the Lemmata}

Proof of Lemma 1: (1) Suppose otherwise, that is for some set of alternatives $\bar{N} \subseteq N$ and some preference profile $D_{\bar{N}} \in \mathcal{D}^{\bar{N}}$, the derived social preference relation $R_{f}\left(D_{\bar{N}}\right)$ is not transitive. Then, there are three alternatives $x, y, z \in K$ such that $x R_{f}\left(D_{\bar{N}}\right) y, y R_{f}\left(D_{\bar{N}}\right) z$, and $z P_{f}\left(D_{\bar{N}}\right) x$. By definition of the derived social welfare function $F_{f}^{\bar{N}}, z P_{f}\left(D_{\bar{N}}\right) x$ is equivalent to $f^{\{x, z\}}\left(D_{\bar{N}}\right)=\{z\}$ and $y R_{f}\left(D_{\bar{N}}\right) z$ implies $f^{\{y, z\}}\left(D_{\bar{N}}\right) \in\{\{y\},\{y, z\}\}$. Now, if $x \in f^{\{x, y, z\}}\left(D_{\bar{N}}\right)$, then $x \in f^{\{x, y, z\}}\left(D_{\bar{N}}\right) \cap\{x, z\}$. Apply the definition of consistency of alternatives to see that $x \in f^{\{x, z\}}\left(D_{\bar{N}}\right)$. This is a contradiction to $f^{\{x, z\}}\left(D_{\bar{N}}\right)=\{z\}$, and therefore, it is possible to conclude that $x \notin f^{\{x, y, z\}}\left(D_{\bar{N}}\right)$ or $f^{\{x, y, z\}}\left(D_{\bar{N}}\right) \in$ $\{\{z\},\{y, z\},\{y\}\}$. If $f^{\{x, y, z\}}\left(D_{\bar{N}}\right)=\{z\}$, then $y \notin f^{\{x, y, z\}}\left(D_{\bar{N}}\right) \cap\{y, z\}$ and $z \in$ $f^{\{x, y, z\}}\left(D_{\bar{N}}\right) \cap\{y, z\}$. Both observations together imply that $f^{\{y, z\}}\left(D_{\bar{N}}\right)=\{z\}$, because the family $\left\{f^{\bar{K}, \bar{N}}\right\}_{\bar{K} \subseteq K, \bar{N} \subseteq N}$ is consistent in alternatives. This is a contradiction to $f^{\{y, z\}}\left(D_{\bar{N}}\right) \in\{\{y\},\{y, z\}\}$, and therefore, $f^{\{x, y, z\}}\left(D_{\bar{N}}\right) \neq\{z\}$. So far, we can deduce that $f^{\{x, y, z\}}\left(D_{\bar{N}}\right) \in\{\{y\},\{y, z\}\}$. Finally, since $y \in$ $f^{\{x, y, z\}}\left(D_{\bar{N}}\right) \cap\{x, y\}$ and $x \notin f^{\{x, y, z\}}\left(D_{\bar{N}}\right) \cap\{x, y\}$, we can apply consistency in alternatives a last time to verify that $f^{\{x, y\}}\left(D_{\bar{N}}\right)=\{y\}$. But this contradiction to $x R_{f}\left(D_{\bar{N}}\right) y$, because $x R_{f}\left(D_{\bar{N}}\right) y$ is equivalent to $f^{\{x, y\}}\left(D_{\bar{N}}\right) \in\{\{x\},\{x, y\}\}$.

(2) We show that given the sets $\bar{K} \subseteq K$ and $\bar{N} \subseteq N$ and the preference profile $D_{\bar{N}} \in \mathcal{D}^{\bar{N}},\left.x \in R_{f}^{1}\left(D_{\bar{N}}\right)\right|_{\bar{K}}$ if and only if $x \in f^{\bar{K}}\left(D_{\bar{N}}\right)$. Suppose that $x \in f^{\bar{K}}\left(D_{\bar{N}}\right)$. Thus, $x \in f^{\bar{K}}\left(D_{\bar{N}}\right) \cap\{x, y\}$ for all $y \in \bar{K}$ and it follows from consistency in alternatives that $x \in f^{\{x, y\}}\left(D_{\bar{N}}\right)$ for all $y \in \bar{K}$. Next, apply the definition of the derived social welfare function $F_{f}^{\bar{N}}$ to see that $x R_{f}\left(D_{\bar{N}}\right) y$ for all $y \in \bar{K}$ which is the same as $\left.x \in R_{f}^{1}\left(D_{\bar{N}}\right)\right|_{\bar{K}}$. To prove the other inclusion suppose that $\left.x \in R_{f}^{1}\left(D_{\bar{N}}\right)\right|_{\bar{K}}$ or $x R_{f}\left(D_{\bar{N}}\right) y$ for all $y \in \bar{K}$. By definition of the derived social welfare function $F_{f}^{\bar{N}}, x \in f^{\{x, y\}}\left(D_{\bar{N}}\right)$ for all $y \in \bar{K}$. To prove that $x \in f^{\bar{K}}\left(D_{\bar{N}}\right)$ we use an induction argument. Let $M \subset \bar{K}$ be such that $x \in M$ and $3 \leq|M|<\bar{k}$. Suppose that for all $\bar{M} \subseteq M$ such that $x \in \bar{M}, x \in f^{\bar{M}}\left(D_{\bar{N}}\right)$. It remains to establish that for all 
$y \in \bar{K} \backslash M, x \in f^{M \cup\{y\}}\left(D_{\bar{N}}\right)$. Suppose contrarily that $x \notin f^{M \cup\{y\}}\left(D_{\bar{N}}\right)$. If $y \in f^{M \cup\{y\}}\left(D_{\bar{N}}\right)$, then $y \in f^{M \cup\{y\}}\left(D_{\bar{N}}\right) \cap\{x, y\}$ and it follows from the definition of consistency in alternatives that $f^{\{x, y\}}\left(D_{\bar{N}}\right)=\{y\}$. But this cannot be, because $x \in f^{\{x, y\}}\left(D_{\bar{N}}\right)$ for all $y \in \bar{K}$ by assumption. On the other hand, if $y \notin f^{M \cup\{y\}}\left(D_{\bar{N}}\right)$, then $f^{M \cup\{y\}}\left(D_{\bar{N}}\right) \subseteq M$. Since it follows from the definition of consistency in alternatives that $f^{M}\left(D_{\bar{N}}\right)=f^{M \cup\{y\}}\left(D_{\bar{N}}\right) \cap M$, the condition $f^{M \cup\{y\}}\left(D_{\bar{N}}\right)=f^{M}\left(D_{\bar{N}}\right)$ has to be satisfied. This is not possible either, because $x \in f^{M}\left(D_{\bar{N}}\right)$ by assumption, and therefore, we conclude that $x \in f^{M \cup\{y\}}\left(D_{\bar{N}}\right)$. For the special case when $M=\bar{K} \backslash\{y\}$ the result reduces to $x \in f^{\bar{K}}\left(D_{\bar{N}}\right)$.

Proof of Lemma 2: It is easy to see that if $f$ depends on the support of the alternatives, then $f$ is strictly symmetric. To show the other inclusion suppose that $f: \mathcal{D}^{N} \rightarrow 2^{K} \backslash\{\emptyset\}$ is strictly symmetric and consider two preference profiles $D, D^{\prime} \in \mathcal{D}^{N}$ which are such that $N_{x}(D)=N_{x}\left(D^{\prime}\right)$ for all $x \in K$. The following algorithm proves by double induction that $f(D)=f\left(D^{\prime}\right)$.

Step 1: In the beginning, define the set $S_{1}$ as $S_{1}=K \backslash\left\{\left\{G\left(D_{1}\right) \cap G\left(D_{1}^{\prime}\right)\right\} \cup\right.$ $\left.\left\{B\left(D_{1}\right) \cap B\left(D_{1}^{\prime}\right)\right\}\right\}$. Notice that $S_{1}$ consists of all alternatives which are differently ordered for individual 1 according to the preference relations $D_{1}$ and $D_{1}^{\prime}$. Let $s_{1}=\left|S_{1}\right| \geq 0$. If $s_{1}=0$, then $D_{1}=D_{1}^{\prime}$. In this case, let $D^{1} \in \mathcal{D}^{N}$ be equal to $D \in \mathcal{D}^{N}$. We conclude that $f\left(D^{1}\right)=f(D)$ and $N_{y}\left(D^{1}\right)=N_{y}(D)$ for all $y \in K$. If $s_{1}>0$, then, without loss of generality, we can order the alternatives in $S_{1}$ according to the one-to-one mapping $g: S_{1} \rightarrow \mathbb{N}_{+}$which is such that $g\left(S_{1}\right)=\left\{1,2, \ldots, s_{1}\right\}\left(g\left(S_{1}\right)\right.$ is the set obtained by applying the mapping $g$ to all elements of $S_{1}$ ). Now proceed to step 1.1 of the algorithm.

Step 1.1: Suppose that $g(x)=1$. If $x \in G\left(D_{1}\right)$ and $x \notin G\left(D_{1}^{\prime}\right)$, then there is an individual $i>1$ such that $x \in G\left(D_{i}^{\prime}\right)$ and $x \notin G\left(D_{i}\right)$, because $N_{y}(D)=N_{y}\left(D^{\prime}\right)$ for all $y \in K$ and in particular for alternative $x \in S_{1}$. Let $1<i \leq n$ be the smallest integer such that $x \in G\left(D_{i}^{\prime}\right)$ and $x \notin G\left(D_{i}\right)$. Next, set the preference profile $D^{1.1}$ equal to $G\left(D_{1}^{1.1}\right)=G\left(D_{1}\right) \backslash\{x\}, G\left(D_{i}^{1.1}\right)=G\left(D_{i}\right) \cup\{x\}$ and $D_{l}^{1.1}=$ $D_{l}$ for all $l \neq 1, i$. Since $f$ is strictly symmetric, $f\left(D^{1.1}\right)=f(D)$. Notice that for all $y \in K, N_{y}\left(D^{1.1}\right)=N_{y}(D)$. On the other hand, if $x \in G\left(D_{1}^{\prime}\right)$ and $x \notin G\left(D_{1}\right)$, 
then there is an individual $j>1$ such that $x \in G\left(D_{j}\right)$ and $x \notin G\left(D_{j}^{\prime}\right)$, because $N_{y}(D)=N_{y}\left(D^{\prime}\right)$ for all $y \in K$ and in particular for alternative $x \in S_{1}$. Let $1<$ $j \leq n$ be the smallest integer such that $x \in G\left(D_{j}\right)$ and $x \notin G\left(D_{j}^{\prime}\right)$. Next, set the preference profile $D^{1.1}$ equal to $G\left(D_{1}^{1.1}\right)=G\left(D_{1}\right) \cup\{x\}, G\left(D_{j}^{1.1}\right)=G\left(D_{j}\right) \backslash\{x\}$ and $D_{l}^{1.1}=D_{l}$ for all $l \neq 1, j$. Since $f$ is strictly symmetric, $f\left(D^{1.1}\right)=f(D)$. Notice that for all $y \in K, N_{y}\left(D^{1.1}\right)=N_{y}(D)$.

Let $M=\{1, \ldots, m\} \subseteq S_{1}, 2 \leq m<s_{1}$, be the set of the first $m$ alternatives of $S_{1}$. Suppose that for all $\bar{M}=\{1, \ldots, \bar{m}\} \subseteq M, f\left(D^{1 \cdot \bar{m}}\right)=f\left(D^{1 \cdot \bar{m}-1}\right)$ and for all $y \in K, N_{y}\left(D^{1 \cdot \bar{m}}\right)=N_{y}\left(D^{1 \cdot \bar{m}-1}\right)$. We show that given the set $M \cup\{m+1\}$, $f\left(D^{1 . m+1}\right)=f\left(D^{1 . m}\right)$ and $N_{y}\left(D^{1 . m+1}\right)=N_{y}\left(D^{1 . m}\right)$ for all $y \in K$.

Step 1.m+1: Suppose that $g(z)=m+1$. If $z \in G\left(D_{1}^{1 . m}\right)$ and $z \notin G\left(D_{1}^{\prime}\right)$, then there is an individual $i>1$ such that $z \in G\left(D_{i}^{\prime}\right)$ and $z \notin G\left(D_{i}^{1 . m}\right)$, because by the induction hypothesis $N_{y}\left(D^{1 \cdot m}\right)=N_{y}\left(D^{\prime}\right)$ for all $y \in K$ and in particular for alternative $z \in S_{1}$. Let $1<i \leq n$ be the smallest integer such that $z \in G\left(D_{i}^{\prime}\right)$ and $z \notin G\left(D_{i}^{1 . m}\right)$. Next, set the preference profile $D^{1 . m+1}$ equal to $G\left(D_{1}^{1 . m+1}\right)=G\left(D_{1}^{1 . m}\right) \backslash\{z\}, G\left(D_{i}^{1 . m+1}\right)=G\left(D_{i}^{1 . m}\right) \cup\{z\}$ and $D_{l}^{1 . m+1}=D_{l}^{1 . m}$ for all $l \neq 1, i$. Since $f$ is strictly symmetric, $f\left(D^{1 . m+1}\right)=f\left(D^{1 . m}\right)$. Notice that for all $y \in K, N_{y}\left(D^{1 . m+1}\right)=N_{y}\left(D^{1 . m}\right)$. On the other hand, if $z \in G\left(D_{1}^{\prime}\right)$ and $z \notin G\left(D_{1}^{1 . m}\right)$, then there is an individual $j>1$ such that $z \in G\left(D_{j}^{1 . m}\right)$ and $z \notin G\left(D_{j}^{\prime}\right)$, because by the induction hypothesis $N_{y}\left(D^{1 . m}\right)=N_{y}\left(D^{\prime}\right)$ for all $y \in K$ and in particular for alternative $z \in S_{1}$. Let $1<j \leq n$ be the smallest integer such that $z \in G\left(D_{j}^{1 . m}\right)$ and $z \notin G\left(D_{j}^{\prime}\right)$. Next, set the preference profile $D^{1 . m+1}$ equal to $G\left(D_{1}^{1 . m+1}\right)=G\left(D_{1}^{1 . m}\right) \cup\{z\}, G\left(D_{j}^{1 . m+1}\right)=G\left(D_{j}^{1 . m}\right) \backslash\{z\}$ and $D_{l}^{1 . m+1}=D_{l}^{1 . m}$ for all $l \neq 1, j$. Since $f$ is strictly symmetric, $f\left(D^{1 . m+1}\right)=$ $f\left(D^{1 . m}\right)$. Notice that for all $y \in K, N_{y}\left(D^{1 \cdot m+1}\right)=N_{y}\left(D^{1 . m}\right)$.

Let $D^{1} \in \mathcal{D}^{N}$ be equal to $D^{1 . s_{1}} \in \mathcal{D}^{N}$. So far it has been shown by induction that at $D^{1}=\left(D_{1}^{\prime}, D_{2}^{1}, \ldots, D_{n}^{1}\right) \in \mathcal{D}^{N}, f\left(D^{1}\right)=f(D)$ and $N_{y}\left(D^{1}\right)=N_{y}(D)$ for all $y \in K$. Let $2 \leq t<n-1$, and, given the integer $t$, define the preference profile $D^{t}$ as $D^{t}=\left(D_{1}^{\prime}, \ldots, D_{t}^{\prime}, D_{t+1}^{t}, \ldots, D_{n}^{t}\right) \in \mathcal{D}^{N}$. Suppose that for all $2 \leq$ $\bar{t} \leq t, f\left(D^{\bar{t}}\right)=f\left(D^{\bar{t}-1}\right)$ and $N_{y}\left(D^{\bar{t}}\right)=N_{y}\left(D^{\bar{t}-1}\right)$ for all $y \in K$. To finish the proof we have to show that $f\left(D^{t+1}\right)=f\left(D^{t}\right)$. 
$\underline{\text { Step t+1: }}$ In the beginning, define the set $S_{t+1}$ as $S_{t+1}=K \backslash\left\{\left\{G\left(D_{t+1}^{t}\right) \cap\right.\right.$ $\left.\left.G\left(D_{t+1}^{\prime}\right)\right\} \cup\left\{B\left(D_{t+1}^{t}\right) \cap B\left(D_{t+1}^{\prime}\right)\right\}\right\}$. Notice that $S_{t+1}$ consists of all alternatives which are ordered differently for individual $t+1$ according to the preference relations $D_{t+1}^{t}$ and $D_{t+1}^{\prime}$. Let $s_{t+1}=\left|S_{t+1}\right| \geq 0$. If $s_{t+1}=0$, then $D_{t+1}^{t}=D_{t+1}^{\prime}$. In this case, let $D^{t+1} \in \mathcal{D}^{N}$ be equal to $D^{t} \in \mathcal{D}^{N}$. We conclude that $f\left(D^{t+1}\right)=$ $f\left(D^{t}\right)$ and $N_{y}\left(D^{t+1}\right)=N_{y}\left(D^{t}\right)$ for all $y \in K$. If $s_{t+1}>0$, then, without loss of generality, we can order the alternatives in $S_{t+1}$ according to the one-to-one mapping $g: S_{t+1} \rightarrow \mathbb{N}_{+}$which is such that $g\left(S_{t+1}\right)=\left\{1,2, \ldots, s_{t+1}\right\}\left(g\left(S_{t+1}\right)\right.$ is the set obtained by applying the mapping $g$ to all elements of $\left.S_{t+1}\right)$. Now proceed to step $t+1.1$ of the algorithm.

Step t+1.1: Suppose that $g(x)=1$. If $x \in G\left(D_{t+1}^{t}\right)$ and $x \notin G\left(D_{t+1}^{\prime}\right)$, then there is an individual $i>t+1$ such that $x \in G\left(D_{i}^{\prime}\right)$ and $x \notin G\left(D_{i}^{t}\right)$, because by the induction hypothesis $N_{y}\left(D^{t}\right)=N_{y}\left(D^{\prime}\right)$ for all $y \in K$ (and in particular for alternative $\left.x \in S_{t+1}\right)$ and $D_{l}^{t}=D_{l}^{\prime}$ for all $l \leq t$. Let $t+1<i \leq n$ be the smallest integer such that $x \in G\left(D_{i}^{\prime}\right)$ and $x \notin G\left(D_{i}^{t}\right)$. Next, set the preference profile $D^{t+1.1}$ equal to $G\left(D_{t+1}^{t+1.1}\right)=G\left(D_{t+1}^{t}\right) \backslash\{x\}, G\left(D_{i}^{t+1.1}\right)=G\left(D_{i}^{t}\right) \cup\{x\}$ and $D_{l}^{t+1.1}=D_{l}^{t}$ for all $l \neq t+1, i$. Since $f$ is strictly symmetric, $f\left(D^{t+1.1}\right)=$ $f\left(D^{t}\right)$. Notice that for all $y \in K, N_{y}\left(D^{t+1.1}\right)=N_{y}\left(D^{t}\right)$. On the other hand, if $x \in G\left(D_{t+1}^{\prime}\right)$ and $x \notin G\left(D_{t+1}^{t}\right)$, then there exists an individual $j>t+1$ such that $x \in G\left(D_{j}^{t}\right)$ and $x \notin G\left(D_{j}^{\prime}\right)$, because by the induction hypothesis $N_{y}\left(D^{t}\right)=N_{y}\left(D^{\prime}\right)$ for all $y \in K$ (and in particular for alternative $x \in S_{t+1}$ ) and $D_{l}^{t}=D_{l}^{\prime}$ for all $l \leq t$. Let $t+1<j \leq n$ be the smallest integer such that $x \in G\left(D_{j}^{t}\right)$ and $x \notin G\left(D_{j}^{\prime}\right)$. Next, set the preference profile $D^{t+1.1}$ equal to $G\left(D_{t+1}^{t+1.1}\right)=G\left(D_{t+1}^{t}\right) \cup\{x\}, G\left(D_{j}^{t+1.1}\right)=G\left(D_{j}^{t}\right) \backslash\{x\}$ and $D_{l}^{t+1.1}=D_{l}^{t}$ for all $l \neq 1, j$. Since $f$ is strictly symmetric, $f\left(D^{t+1.1}\right)=f\left(D^{t}\right)$. Notice that for all $y \in K, N_{y}\left(D^{t+1.1}\right)=N_{y}\left(D^{t}\right)$.

Let $M=\{1, \ldots, m\} \subseteq S_{t+1}, 2 \leq m<s_{t+1}$, be the set of the first $m$ alternatives of $S_{t+1}$. Suppose that for all $\bar{M}=\{1, \ldots, \bar{m}\} \subseteq M, f\left(D^{t+1 . \bar{m}}\right)=$ $f\left(D^{t+1 \cdot \bar{m}-1}\right)$ and for all $y \in K, N_{y}\left(D^{t+1 \cdot \bar{m}}\right)=N_{y}\left(D^{t+1 \cdot \bar{m}-1}\right)$. We show that given the set $M \cup\{m+1\}, f\left(D^{t+1 . m+1}\right)=f\left(D^{t+1 . m}\right)$ and $N_{y}\left(D^{t+1 . m+1}\right)=$ $N_{y}\left(D^{t+1 . m}\right)$ for all $y \in K$. 


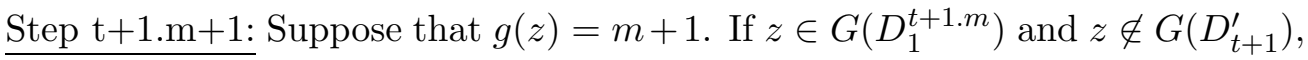
then there is an individual $i>t+1$ such that $z \in G\left(D_{i}^{\prime}\right)$ and $z \notin G\left(D_{i}^{t+1 . m}\right)$, because by the induction hypothesis $N_{y}\left(D^{t+1 . m}\right)=N_{y}\left(D^{\prime}\right)$ for all $y \in K$ (and in particular for alternative $\left.z \in S_{t+1}\right)$ and $D_{l}^{t}=D_{l}^{\prime}$ for all $l \leq t$. Let $t+1<$ $i \leq n$ be the smallest integer such that $z \in G\left(D_{i}^{\prime}\right)$ and $z \notin G\left(D_{i}^{t+1 . m}\right)$. Next, set the preference profile $D^{t+1 . m+1}$ equal to $G\left(D_{1}^{t+1 . m+1}\right)=G\left(D_{1}^{t+1 . m}\right) \backslash\{z\}$, $G\left(D_{i}^{t+1 . m+1}\right)=G\left(D_{i}^{t+1 . m}\right) \cup\{z\}$ and $D_{l}^{t+1 . m+1}=D_{l}^{t+1 . m}$ for all $l \neq 1$, $i$. Since $f$ is strictly symmetric, $f\left(D^{t+1 \cdot m+1}\right)=f\left(D^{t+1 . m}\right)$. Notice that for all $y \in K$, $N_{y}\left(D^{t+1 . m+1}\right)=N_{y}\left(D^{t+1 . m}\right)$. On the other hand, if $z \in G\left(D_{t+1}^{\prime}\right)$ and $z \notin$ $G\left(D_{1}^{t+1 . m}\right)$, then there is an individual $j>t+1$ such that $z \in G\left(D_{j}^{t+1 . m}\right)$ and $z \notin G\left(D_{j}^{\prime}\right)$, because by the induction hypothesis $N_{y}\left(D^{t+1 . m}\right)=N_{y}\left(D^{\prime}\right)$ for all $y \in K$ (and in particular for alternative $z \in S_{t+1}$ ) and $D_{l}^{t+1 . m+1}=D_{l}^{t+1 . m}$ for all $l \neq 1, i$. Let $t+1<j \leq n$ be the smallest integer such that $z \in G\left(D_{j}^{t+1 . m}\right)$ and $z \notin G\left(D_{j}^{\prime}\right)$. Next, set the preference profile $D^{t+1 . m+1}$ equal to $G\left(D_{1}^{t+1 . m+1}\right)=$ $G\left(D_{1}^{t+1 . m}\right) \cup\{z\}, G\left(D_{j}^{t+1 . m+1}\right)=G\left(D_{j}^{t+1 . m}\right) \backslash\{z\}$ and $D_{l}^{t+1 . m+1}=D_{l}^{t+1 . m}$ for all $l \neq 1, j$. Since $f$ is strictly symmetric, $f\left(D^{t+1 . m+1}\right)=f\left(D^{t+1 . m}\right)$. Notice that for all $y \in K, N_{y}\left(D^{t+1 . m+1}\right)=N_{y}\left(D^{t+1 . m}\right)$.

The algorithm finishes after $n-1$ steps, because the conditions $D_{i}^{n-1}=D_{i}^{\prime}$ for all $i \neq n$ and $N_{y}\left(D^{n-1}\right)=N_{y}\left(D^{\prime}\right)$ for all $y \in K$ imply that $S_{n}=\emptyset$.

Proof of Lemma 3: Suppose otherwise. Then, there is a preference profile $D \in \mathcal{D}^{N}$ which is such that $N_{x}(D)=N_{y}(D)$ for some pair of alternatives $(x, y)$, but the social choice function $f$ satisfies $x \in f(D)$ and $y \notin f(D)$. Define the permutation $\mu: K \rightarrow K$ as $\mu(x)=y, \mu(y)=x$, and $\mu(z)=z$ for all $z \in K \backslash\{x, y\}$. Then $f(\mu(D))=f(D)$, because $N_{z}(\mu(D))=N_{z}(D)$ for all $z \in K$ and the social choice function $f$ depends on the support of the alternatives by Lemma 2. Neutrality of $f$ implies that $\mu(f(D))=f(\mu(D))$, and therefore, the condition $\mu(f(D))=f(D)$ has to be satisfied. But if the mapping $\mu$ is applied to the set $f(D)$, then $\mu(x)=y \in \mu(f(D))$. Since $y \notin f(D)$ by assumption, $\mu(f(D)) \neq f(D)$. This is a contradiction. 


\section{An Example of the Algorithm Applied in Lemma 2}

Let $N=\{1,2,3\}$ and $K=\{x, y, z\}$. The preference profile $D \in \mathcal{D}^{N}$ is given by $G\left(D_{1}\right)=\{x, y\}, G\left(D_{2}\right)=\{z\}$, and $G\left(D_{3}\right)=\{x\}$, whereas the preference profile $D^{\prime} \in \mathcal{D}^{N}$ is such that $G\left(D_{1}^{\prime}\right)=G\left(D_{2}^{\prime}\right)=\{x\}$ and $G\left(D_{3}^{\prime}\right)=\{y, z\}$. Observe that $N_{x}(D)=N_{x}\left(D^{\prime}\right)=2$ and $N_{y}(D)=N_{y}\left(D^{\prime}\right)=N_{z}(D)=N_{z}\left(D^{\prime}\right)=1$.

Step 1.1: The set $S_{1}$ consists of all alternatives which are differently ordered for individual 1 according to the preference relations $D_{1}$ and $D_{1}^{\prime}$. Hence, $S_{1}=\{y\}$. Since $y \in G\left(D_{1}\right)$ and $y \notin G\left(D_{1}^{\prime}\right)$, one has to look for individual $i>1$ such that $y \notin G\left(D_{i}\right)$ and $y \in G\left(D_{i}^{\prime}\right)$. This is individual 3. Define $D^{1.1} \in \mathcal{D}^{N}$ as $G\left(D_{1}^{1.1}\right)=G\left(D_{1}\right) \backslash\{y\}=\{x\}, D_{2}^{1.1}=D_{2}$, and $G\left(D_{3}^{1.1}\right)=G\left(D_{3}\right) \cup\{y\}=$ $\{x, y\}$. By strict symmetry, $f\left(D^{1}\right)=f(D)$. Finally, define $D^{1}=D^{1.1}=$ $\left(D_{1}^{\prime}, D_{2}^{1.1}, D_{3}^{1.1}\right)$.

Step 2.1: The set $S_{2}$ consists of all alternatives which are differently ordered for individual 2 according to the preference relations $D_{2}^{1}$ and $D_{2}^{\prime}$. Hence, $S_{2}=$ $\{x, z\}$. Without loss of generality let $g(x)=2$ and $g(z)=1$. Since $z \in G\left(D_{2}^{1}\right)$ and $z \notin G\left(D_{2}^{\prime}\right)$, one has to find an individual $i>2$ such that $z \notin G\left(D_{i}^{1}\right)$ and $z \in G\left(D_{i}^{\prime}\right)$. This is individual 3. Define $D^{2.1} \in \mathcal{D}^{N}$ as $D_{1}^{2.1}=D_{1}^{\prime}$, $G\left(D_{2}^{2.1}\right)=G\left(D_{2}^{1}\right) \backslash\{z\}=\emptyset$ and $G\left(D_{3}^{2.1}\right)=G\left(D_{3}^{1}\right) \cup\{z\}=\{x, y, z\}$. By strict symmetry, $f\left(D^{2.1}\right)=f\left(D^{1}\right)$.

Step 2.2 Finally, consider alternative $x \in S_{2}$. Since $x \notin G\left(D_{2}^{2.1}\right)$ and $x \in$ $G\left(D_{2}^{\prime}\right)$, one has to find individual $j>2$ such that $x \in G\left(D_{j}^{2.1}\right)$ and $x \notin$ $G\left(D_{j}^{\prime}\right)$. This is individual 3. Define $D^{2.2} \in \mathcal{D}^{N}$ as $D_{1}^{2.2}=D_{1}^{\prime}, G\left(D_{2}^{2.2}\right)=$ $G\left(D_{2}^{2.1}\right) \cup\{x\}=\{x\}$ and $G\left(D_{3}^{2.2}\right)=G\left(D_{3}^{2.1}\right) \backslash\{x\}=\{y, z\}$. By strict symmetry, $f\left(D^{2.2}\right)=f\left(D^{2.1}\right)$. The algorithm stops, because $D^{2.2}=D^{\prime}$.

\section{Tightness of Theorem 1}

We exhibit four social choice rules different from Approval Voting which violate one property each.

\section{Strategy-Proofness:}


Due to Vorsatz [16] the Borda Count is equivalent to Approval Voting on the dichotomous preference domain. Since all scoring rules are anonymous, neutral and strictly monotone, any scoring rule different from the Borda Count must be manipulable on the cohesive dichotomous domain according to Theorem 1 . A direct proof of this result can also be found in Vorsatz [16].

\section{Neutrality:}

Let the family of social choice functions $\left\{f_{1}^{\bar{K}, \bar{N}}: \mathcal{D}^{\bar{N}} \rightarrow 2^{K} \backslash\{\emptyset\}\right\}_{\bar{K} \subseteq K, \bar{N} \subseteq N}$ be such that for all $\bar{K} \subseteq K, \bar{N} \subseteq N$ and $D_{\bar{N}} \in \mathcal{D}^{\bar{N}}, f_{1}^{\bar{K}}\left(D_{\bar{N}}\right)=\{x\}$ whenever $x \in f_{A}^{\bar{K}}\left(D_{\bar{N}}\right)$. Otherwise, $f_{1}^{\bar{K}}\left(D_{\bar{N}}\right)=f_{A}^{\bar{K}}\left(D_{\bar{N}}\right)$. The social choice rule $\left\{f_{1}^{\bar{K}, \bar{N}}\right\}_{\bar{K} \subseteq K, \bar{N} \subseteq N}$ is anonymous, strictly monotone and strategy-proof. To see that $f_{1}^{\bar{K}, \bar{N}}$ is not neutral for some $\bar{K}$ and $\bar{N}$ consider the following example.

Let $K=\{x, y\}$ and $N=\{1,2\}$. The preference profile $D \in \mathcal{D}^{N}$ is such that $G\left(D_{1}\right)=G\left(D_{2}\right)=\{x, y\}$. Then, $f_{1}^{\bar{K}}\left(D_{\bar{N}}\right)=\{x\}$. Define the permutation $\mu$ of $K$ as $\mu(x)=y$ and $\mu(y)=x$. Then, $\mu\left(f_{1}^{\bar{K}}\left(D_{\bar{N}}\right)\right)=\{y\}$. Since $\mu\left(D_{\bar{N}}\right)=D_{\bar{N}}$, it has to be that $f_{1}^{\bar{K}}\left(\mu\left(D_{\bar{N}}\right)\right)=\{x\}$. This contradicts neutrality.

\section{Anonymity:}

Let the family of social choice functions $\left\{f_{2}^{\bar{K}, \bar{N}}: \mathcal{D}^{\bar{N}} \rightarrow 2^{\bar{K}} \backslash\{\emptyset\}\right\}_{\bar{K} \subseteq K, \bar{N} \subseteq N}$ be such that for all $\bar{K} \subseteq K, \bar{N} \subseteq N$ and $D_{\bar{N}} \in \mathcal{D}^{\bar{N}}$, if the conditions $\{1\} \in \bar{N}$, $0<g\left(D_{1}\right)<\bar{k}$, and $G\left(D_{1}\right) \cap f_{A}^{\bar{K}}\left(D_{\bar{N}}\right) \neq \emptyset$ are satisfies, then $f_{2}^{\bar{K}}\left(D_{\bar{N}}\right)=$ $G\left(D_{1}\right) \cap f_{A}^{\bar{K}}\left(D_{\bar{N}}\right)$. Otherwise, $f_{2}^{\bar{K}}\left(D_{\bar{N}}\right)=f_{A}^{\bar{K}}\left(D_{\bar{N}}\right)$. The social choice rule $\left\{f_{2}^{\bar{K}, \bar{N}}\right\}_{\bar{K} \subseteq K, \bar{N} \subseteq N}$ is strategy-proof, strictly monotone and neutral. The following example shows that $f_{2}^{\bar{K}, \bar{N}}$ is not anonymous for some $\bar{K} \subseteq K$ and $\bar{N} \subseteq K$.

Let $K=\{x, y\}$ and $N=\{1,2\}$. Suppose that $D \in \mathcal{D}^{N}$ is equal to $G\left(D_{1}\right)=$ $\{x\}$ and $G\left(D_{2}\right)=\{y\}$. Then, $f_{2}(D)=\{x\}$. Define the permutation $\sigma$ of $N$ as $\sigma(1)=2$ and $\sigma(2)=1$. Then, $f_{2}\left(D_{\sigma(N)}\right)=\{y\}$. This contradicts anonymity.

\section{Strict Monotonicity:}

Given $D_{\bar{N}} \in \mathcal{D}^{\bar{N}}$, let $C \subseteq \bar{N}$ be such that $i \in C$ if and only if $0<g\left(D_{i}\right)<\bar{k}$. Define the family of social choice functions $\left\{f_{3}^{\bar{K}, \bar{N}}: \mathcal{D}^{\bar{N}} \rightarrow 2^{\bar{K}} \backslash\{\emptyset\}\right\}_{\bar{K} \subseteq K, \bar{N} \subseteq N}$ as follows: For all $\bar{K} \subseteq K, \bar{N} \subseteq N$ and $D_{\bar{N}} \in \mathcal{D}^{\bar{N}}$, (a) $x \in f_{3}^{\bar{K}}\left(D_{\bar{N}}\right)$ if and only if $N_{x}\left(\left.D_{\bar{N}}\right|_{C}\right) \geq 1$ and $C \neq \emptyset$, (b) if there does not exist a $x \in \bar{K}$ 
such that $N_{x}\left(\left.D_{\bar{N}}\right|_{C}\right) \geq 1$ or $C=\emptyset$, then $f_{3}^{\bar{K}}\left(D_{\bar{N}}\right)=\bar{K}$. The social choice rule $\left\{f_{3}^{\bar{K}, \bar{N}}\right\}_{\bar{K} \subseteq K, \bar{N} \subseteq N}$ is strategy-proof, neutral and anonymous. The next example shows that $\left\{f_{3}^{\overline{\bar{K}}, \bar{N}}\right\}_{\bar{K} \subset K, \bar{N} \subset N}$ is not strictly monotone.

Suppose that $K=\{x, y, z\}$ and $N=\{1,2\}$. Let $D, D^{\prime} \in \mathcal{D}^{N}$ be such that $G\left(D_{1}\right)=\{y\}, G\left(D_{1}^{\prime}\right)=\{x, y\}$ and $G\left(D_{2}\right)=G\left(D_{2}^{\prime}\right)=\{x\}$. Then, $C=$ $C^{\prime}=\{1,2\}$ which implies that $f_{3}(D)=f_{3}\left(D^{\prime}\right)=\{x, y\}$. This contradicts strict monotonicity.

\section{Tightness of Theorem 2 and 3}

We exhibit three social choice functions which violate in each Theorem one property each.

\section{Efficiency (Theorem 3) and Strict Monotonicity (Theorem 2):}

Let $g: \mathcal{D}^{N} \rightarrow 2^{K} \backslash\{\emptyset\}$ be as follows: For all $D \in \mathcal{D}^{N}$, (a) for all $x \in K, x \in g(D)$ if and only if $N_{x}(D) \geq 1$, and (b) if there does not exist any alternative $x \in K$ such that $N_{x}(D) \geq 1$, then $f(D)=K$. The social choice function $g$ is strictly symmetric and neutral. To see that $g$ is neither strictly monotone nor efficient consider the following example.

Let $K=\{x, y, z\}$ and $N=\{1,2,3\}$. The preference profiles $D, D^{\prime} \in \mathcal{D}^{N}$ are such that $G\left(D_{1}\right)=G\left(D_{1}^{\prime}\right)=\{x\}, G\left(D_{2}\right)=\{y\}, G\left(D_{2}^{\prime}\right)=\{y, z\}$ and $G\left(D_{3}\right)=$ $G\left(D_{3}^{\prime}\right)=\{x, y, z\}$. Hence, $g(D)=g\left(D^{\prime}\right)=\{x, y, z\}$. Since $\{x, y\} \succ_{D_{1}}\{x, y, z\}$, $\{x, y\} \succ_{D_{2}}\{x, y, z\}$, and $\{x, y\} \sim_{D_{3}}\{x, y, z\}, g$ is not efficient. Moreover, if $g$ was strictly monotone, then it would be the case that $g\left(D^{\prime}\right)=\{z\}$.

\section{Neutrality (Theorem 2 and 3):}

The non-neutral social choice function $f_{1}$ is strictly symmetric, strictly monotone and efficient.

\section{Strict Symmetry (Theorem 2 and 3):}

Given $D \in \mathcal{D}^{N}$, the set of individuals with the largest set of good alternatives is defined as $G_{\max }(D)=\left\{i \in N\right.$ : for all $\left.j \in N,\left|G\left(D_{i}\right)\right| \geq\left|G\left(D_{j}\right)\right|\right\}$. Let $h: \mathcal{D}^{N} \rightarrow 2^{K} \backslash\{\emptyset\}$ be as follows: For all $D \in \mathcal{D}^{N}$, if $\bigcap_{i \in G_{\max }(D)} G\left(D_{i}\right) \cap f_{A}(D) \neq \emptyset$, then $h(D)=\bigcap_{i \in G_{\max }(D)} G\left(D_{i}\right) \cap f_{A}(D)$; otherwise, $h(D)=f_{A}(D)$. The social 
choice function $h$ is neutral, efficient and strictly monotone (it is anonymous, too). To see that $h$ is not strictly symmetric consider the following example.

Let $K=\{x, y, z\}$ and $N=\{1,2\}$. Suppose that $D \in \mathcal{D}^{N}$ is such that $G\left(D_{1}\right)=\{x, y, z\}$ and $G\left(D_{2}\right)=\emptyset$. Then, $h(D)=G\left(D_{1}\right) \cap f_{A}(D)=K$. Consider the preference profile $D^{\prime} \in \mathcal{D}^{N}$ which is such that $G\left(D_{1}^{\prime}\right)=\{x, y\}$ and $G\left(D_{2}^{\prime}\right)=\{z\}$. Then, $h\left(D^{\prime}\right)=G\left(D_{1}^{\prime}\right) \cap f_{A}\left(D^{\prime}\right)=\{x, y\}$. Since $h(D) \neq h\left(D^{\prime}\right)$, the social choice function $h$ is not strictly symmetric. 